\title{
Optionen zur Intensivierung einer Basalinsulintherapie bei Menschen mit Typ-2-Diabetes
}

\section{Options for Intensifying a Basal Insulin Therapy in Humans with Type 2 Diabetes}

Autoren

Institute
B Gallwitz ${ }^{1}$, S Matthaei ${ }^{2}$

Universitätsklinikum Tübingen, Medizinische Klinik IV, Tübingen

Diabetes-Zentrum Quakenbrück, Fachabteilung für Diabetologie, Endokrinologie und Stoffwechsel am Christlichen Krankenhaus Quakenbrück, Quakenbrück
Schlüsselwörter

- Typ-2-Diabetes

- Insulin

- Exenatid

- Liraglutid

- Albiglutid

- Hypoglykämien

- Körpergewicht

- GLP-1-Rezeptoragonist

Key words

- type 2 diabetes

- insulin

- exenatide

- liraglutide

- albiglutide

- hypoglycaemia

- body weight

- GLP-1-receptor agonist

\section{Bibliografie}

DOI http://dx.doi.org/

10.1055/s-0035-1553048

Diabetologie 2015; 10 :

129-140 ๔ Georg Thieme

Verlag KG Stuttgart · New York .

ISSN 1861-9002

Korrespondenzadresse

Prof. Dr. med. Baptist Gallwitz

Universitätsklinikum Tübingen,

Medizinische Klinik IV

Otfried-Müller-Str. 10

72076 Tübingen

Tel.: 070712982093 ,

baptist.gallwitz@med.uni-

tuebingen.de

\section{Zusammenfassung \\ $\nabla$}

Einleitung: Bei Menschen mit Typ-2-Diabetes, die eine mit Basalinsulin unterstützte orale Therapie (BOT) erhalten, entsteht häufig die Notwendigkeit zur weiteren Intensivierung der Therapie. In derartigen Situationen sind Glucagon-like-peptide1-Rezeptoragonisten (GLP-1-RA) wegen ihrer sich ergänzenden Eigenschaften zu Insulin besonders gut für die Kombinationstherapie geeignet. Methodik: Anhand von Studien zur Intensivierung einer bestehenden Insulintherapie mit den GLP-1-RA Exenatid, Liraglutid oder Albiglutid wird der Stellenwert von GLP-1-RA im Vergleich zu kurz wirksamen Insulinanaloga bei der Intensivierung einer BOT bei Typ-2-Diabetes analysiert. Ergebnisse: In der Gesamtschau von zehn randomisierten kontrollierten Studien, insbesondere der 4B-Studie zu Exenatid, der BEGIN-VICTOZAADD-ON-Studie zu Liraglutid und der HARMONY-6-Studie zu Albiglutid, wurden mit GLP-1RA und kurz wirksamen Insulinen mindestens gleich gute $\mathrm{HbA}_{1 \mathrm{c}}$-Reduktionen erreicht. Einzelne Studien zeigen für GLP-1-RA Vorteile hinsichtlich einer um ca. 0,5-1,5 mmol/l stärkeren Reduktion des Nüchternblutzuckers, weniger Hypoglykämien, einer deutlich günstigeren Gewichtsentwicklung, einer Reduktion des systolischen Blutdrucks um ca. 4-5 mmHg. Dagegen können mit kurz wirksamen Insulinanaloga postprandiale Blutzuckerwerte gezielter beeinflusst und zur Förderung der Therapieadhärenz und -persistenz unerwünschte gastrointestinale Ereignisse zu Therapiebeginn vermieden werden. GLP-1-RA können die Therapieadhärenz im Vergleich zu kurz wirksamen Insulinanaloga verbessern durch Körpergewichtsreduktionen, weniger Hypoglykämien, eine geringe Komplexität der Behandlung und eine höhere Therapiezufriedenheit. Bei bestimmten Patienten mit Typ-2Diabetes konnte im Verlauf die Insulindosis reduziert oder Insulin abgesetzt werden.

\section{Abstract \\ $\nabla$}

Introduction: People with type 2 diabetes receiving a basal insulin therapy in combination with oral antidiabetic drugs (BOT) often develop the need for further intensification of treatment. In such situations, Glucagon-like peptide-1 receptor agonists (GLP-1-RA) are particularly suited for a combination treatment because their properties are complementary supportive to insulin.

Methods: The effect of the GLP-1 RA exenatide, liraglutide and albiglutide is analysed and compared with short-acting insulin analogues during intensification of BOT in patients with type $2 \mathrm{di}-$ abetes based on trials assessing the intensification of an existing insulin therapy.

Results: GLP-1 RA and short-acting insulins achieved at least equivalent $\mathrm{HbA}_{1 \mathrm{c}}$ reductions in the review of ten randomised controlled trials, especially the $4 \mathrm{~B}$ trial on exenatide, the BEGIN VICTOZA ADD-ON trial on liraglutide, and the HARMONY 6 trial on albiglutide. Individual trials showed advantages for GLP-1 RA in terms of an approx. $0.5-1.5 \mathrm{mmol} / \mathrm{l}$ greater reduction of fasting blood glucose, less episodes of hypoglycaemia, a clearly more favourable weight development, a reduction of systolic blood pressure by approx. 4-5 mmHg. On the other hand, short-acting insulin analogues are able to affect postprandial blood glucose values more specifically and to avoid adverse gastrointestinal events at the start of treatment if compared to GLP-1 RA which helps to promote treatment adherence and persistence. GLP-1 RA can help to improve treatment adherence compared with short-acting insulin analogues by body weight reduction, less episodes of hypoglycaemia, a lower complexity of treatment as well as a higher treatment satisfaction. Specific patients with type 2 diabetes were able to reduce the insulin dose or discontinue insulin completely in the course of treatment. 
Schlussfolgerung: Der Vergleich von GLP-1-RA und kurz wirksamen Insulinanaloga als Optionen zur Therapieintensivierung bei einer BOT ergibt ein differenziertes Bild, das die Basis für ein individualisiertes therapeutisches Vorgehen bei Patienten mit unzureichend kontrolliertem Typ-2-Diabetes darstellt. Offene Fragen bestehen hinsichtlich langfristiger Auswirkungen auf diabetesbezogene Komplikationen und bezüglich des individuell optimalen Zeitpunktes der erstmaligen Gabe von GLP-1-RA.

\section{Einleitung}

Vorrangiges Ziel der Therapie von Menschen mit Typ-2-Diabetes ist neben der Senkung der Rate der diabetesbedingten Komplikationen die Verbesserung der Lebensqualität, der Morbidität sowie der Mortalität. Fortschritte im Umgang mit der Erkrankung ergeben sich nicht nur durch neue Antidiabetika, sondern insbesondere auch aus der Individualisierung von Behandlungszielen einschließlich der Abkehr von starren $\mathrm{HbA}_{1 \mathrm{c}}$-Zielwerten. Im Einzelfall sind Begleitumstände zu berücksichtigen wie das Verhalten des Patienten (Therapietreue, Motivation, Selbständigkeit), die Diabetesdauer, die Lebenserwartung, wichtige Begleiterkrankungen, Gefäßkomplikationen, Hypoglykämierisiken bzw. Risiken durch andere Nebenwirkungen und Ressourcen bzw. die Unterstützung anderer Art (Inzucchi 2015). Zudem sollte das Körpergewicht beachtet werden. Der Therapiealgorithmus zum Typ-2-Diabetes ist ein Spiegelbild der zunehmenden Komplexität der Behandlungsoptionen, die sich auch in der neuen Nationalen VersorgungsLeitlinie zur Therapie des Typ-2-Diabetes zeigt (Landgraf 2013). Daher erscheint es sinnvoll, auch Einzelfragestellungen innerhalb des Therapiealgorithmus zu betrachten.

Eine Frage mit gewisser Aktualität betrifft Patienten, die bereits eine mit Basalinsulin unterstützte orale Therapie (BOT) erhalten und bei denen eine Intensivierung der antidiabetischen Therapie mit einem weiteren subkutan zu applizierenden Antidiabetikum in Erwägung gezogen wird. Für derartige Situationen analysiert die hier vorliegende Übersicht den Stellenwert der Glucagonlike-peptide-1 (GLP-1)-Rezeptoragonisten (GLP-1-RA) im Vergleich zu kurz wirksamen Insulinanaloga.

\section{Inkretin-basierte Therapie}

$\nabla$

GLP-1 gehört zu den Inkretin-Hormonen und wird nach der Nahrungsaufnahme freigesetzt. In der Folge wird die Insulinsekretion glucoseabhängig stimuliert sowie die Glucagonsekretion gehemmt (Drucker 2006). Dieser kombinierte Effekt auf die Inselzellen des Pankreas ist ein wichtiger Bestandteil der antidiabetischen Wirksamkeit einer Inkretin-basierten Therapie. GLP-1-Rezeptoren befinden sich auch in Zellen außerhalb des Pankreas, so dass GLP-1 beispielsweise eine Verzögerung der Magenentleerung (Nauck 1997), ein über den Hypothalamus vermitteltes Sättigungsgefühl (Gutzwiller 1999) und kardiovaskuläre Effekte (Okerson 2012) vermitteln kann.

Eine Inkretin-basierte Therapie kann mit subkutan zu verabreichenden GLP-1-RA oder mit den für die orale Applikation verfügbaren DPP-4-Inhibitoren durchgeführt werden (Ahrén 2014). GLP-1-RA sind Analoga zum GLP-1 und haben dessen physiologische Wirkungen an GLP-1-Rezeptoren. Dagegen erhöhen DPP-4Inhibitoren die GLP-1-Spiegel, indem sie das Enzym DPP-4 kompetitiv hemmen und dadurch die Inaktivierung der endogenen
Conclusion: The comparison of GLP-1 RA and short-acting insulin analogues as options of treatment intensification in patients on BOT gives a differentiated picture, which is the basis for an individualised therapeutic procedure in patients with inadequate control of their type 2 diabetes. There are open questions regarding the long-term effects of diabetes-related complications and regarding the optimal individual point of time of the first GLP-1 RA administration.

Inkretin-Hormone GLP-1 und GIP (glucose-dependent insulinotropic polypeptide) verlangsamen (Brown 2012).

Wenngleich beide Inkretin-basierten Therapien als vorteilhaft hinsichtlich der Hypoglykämierisiken gelten, wurden mit GLP-1RA eine stärkere $\mathrm{HbA}_{1 \mathrm{c}}$-Reduktionen erreicht als mit DPP-4-Inhibitoren (Pratley 2010, Bergenstal 2010). Zudem erscheinen GLP1-RA wegen der gezeigten stärkeren Reduktionen des Körpergewichts (Pratley 2010, Bergenstal 2010) besonders gut für eine Kombination mit Basalinsulin geeignet, da unter einer Insulintherapie häufig eine Gewichtszunahme zu beobachten ist.

Aktuell breit im deutschsprachigen Raum angewendet und unter anderem auch für die Kombination mit Basalinsulinen zugelassen sind ein GLP-1-RA mit kürzerer Halbwertszeit (HWZ) (Exenatid; Byetta ${ }^{\circledR}$, AstraZeneca GmbH, 22876 Wedel, Deutschland) und ein GLP-1-RA mit längerer HWZ (Liraglutid; Victoza ${ }^{\circledR}$, Novo Nordisk A/S, Dänemark). Später wurde ein einmal wöchentlich zu applizierender GLP-1-RA unter anderem auch für die Kombinationstherapie mit Basalinsulin zugelassen (Albiglutid; Eperzan $^{\circledR}$, GlaxoSmithKline GmbH \& Co. KG, 80700 München). Da die Exenatid-Depot-Injektionssuspension mit verlängerter Freisetzung (Bydureon ${ }^{\circledR}$, AstraZeneca GmbH, 22876 Wedel, Deutschland) in Europa für die Kombination mit Insulinen nicht zugelassen ist (Fachinformation Bydureon ${ }^{\circledR}$ ), der GLP-1-RA Lixisenatid mit kürzerer HWZ seitens des Herstellers wegen fehlender Preiseinigung im Rahmen des Arzneimittelmarktneuordnungsgesetzes (AMNOG) vom deutschen Markt zurückgezogen wurde und zum kürzlich zugelassenen GLP-1-RA Dulaglutid keine Studienergebnisse zur Kombination mit einem Basalinsulin vorliegen (Thompson 2015), werden diese drei Präparate in dieser Übersicht nicht näher analysiert.

\section{Vorteile der GLP-1-RA}

$\nabla$

GLP-1-RA können auf vielfältige Weise Einfluss auf Stoffwechselparameter von Patienten mit Typ-2-Diabetes nehmen. So wird der Nüchternblutzucker über eine Reduktion der basalen endogenen Glucoseproduktion gesenkt, die bei Patienten mit Typ-2Diabetes durch eine inadäquat vermehrte Glucagonproduktion erhöht ist (Meier 2012, Flint 2011). Die Verbesserung hinsichtlich des postprandialen Blutzuckeranstiegs gelingt nicht nur über die Steigerung der glucoseabhängigen Insulinsekretion, sondern auch über die geringere Aufnahme der oral zugeführten Kohlenhydrate in die Blutzirkulation im Zusammenhang mit einer verlangsamten Magenentleerung (Linnebjerg 2008, Meier 2012, Flint 2011). Die Induktion eines über den Hypothalamus vermittelten Sättigungsgefühls und das geringere Hypoglykämierisiko tragen zur Gewichtsreduktion bei (Ahrén 2014). Außerdem wurden blutdrucksenkende Effekte und möglicherweise positive Einflüsse auf den Lipidstoffwechsel beobachtet (Okerson 2012, Buse 2009). 


\section{Blutdrucksenkende Effekte der GLP-1-RA}

$\nabla$

GLP-1-Rezeptoren wurden auch im Myokard, in glatten Muskelzellen von Koronararterien, in anderen Blutgefäßen sowie in den Gefäßen der Niere gefunden (Fonseca 2014, Okerson 2012). Daher sind kardiovaskuläre Effekte von GLP-1-RA denkbar. Als Klasseneffekt der GLP-1-RA wurde eine Blutdrucksenkung nachgewiesen (Buse 2009, Fonseca 2014, Wang 2013, Seufert und Gallwitz 2014). So reduzierte sich der systolische Blutdruck im Vergleich zu Placebo sowohl bei Exenatid als auch bei Liraglutid im Mittel um ca. $5 \mathrm{mmHg}$ (Wang 2013). Allerdings wurde in einer Untersuchung bei Patienten mit Typ-2-Diabetes und arterieller Hypertonie in den ersten Tagen nach Therapieeinleitung mit Liraglutid $0,6 \mathrm{mg}$ zunächst ein initialer Anstieg des systolischen Blutdrucks beobachtet (von Scholten 2014). Am Tag 29 war dann eine Reduktion um $7 \mathrm{mmHg}$ gegenüber dem Ausgangswert zu verzeichnen $(p=0,11)$. Zugleich wurde ein Abfall des extrazellulären Volumens um 2,0 Liter (95\% CI: 1,0 - 3,1 Liter; p < 0,001) und des „midregional pro-atrial natriuretic peptide“ (MR-proANP) um 20\% (95\% CI: $12-28 \%$; p 0,001) festgestellt.

In Studien zu Liraglutid wurden Blutdruckreduktionen begleitet von einem Pulsanstieg um durchschnittlich 3,22 $\pm 0,45$ bzw. $3,46 \pm 0,39$ pro Minute im Vergleich zu 0,89 $\pm 0,50$ pro Minute in den Placebogruppen (Fonseca 2014). Bei einer längeren Beobachtungszeit der Liraglutid-Therapie von 104 Wochen zeigte sich jedoch eine Verringerung dieses Pulsanstiegs (Astrup 2012).

Der blutdrucksenkende Effekt von GLP-1-RA ist möglicherweise auf eine gesteigerte Natriurese und/oder eine Vasodilatation zurückzuführen (Fonseca 2014). Inwieweit das langfristige kardiovaskuläre Outcome mit GLP-1-RA verbessert werden kann, ist Gegenstand aktueller Studien. Tierversuche und Ergebnisse aus klinischen Studien zu Surrogatparametern weisen auf kardioprotektive Effekte von GLP-1-RA hin (Wu 2011, Seufert und Gallwitz 2014). Eine Metaanalyse zu 14 Studien mit 415 Patienten ergab eine signifikante Verbesserung von regionalen linksventrikulären kontraktilen Parametern unter GLP-1-RA (Liu 2014).

\section{Gastrointestinale Nebenwirkungen der GLP-1-RA $\nabla$}

Gemäß einer Metaanalyse waren in randomisierten kontrollierten Studien in Abhängigkeit von Dosis und Art des GLP-1-RA durchschnittlich zwischen $12 \%$ und $37 \%$ der Patienten von Übelkeit betroffen, im Vergleich zu durchschnittlich 9\% in den Placebo-Gruppen und $4 \%$ in den konventionell behandelten Vergleichsgruppen (Sun 2012). Erbrechen (zwischen $8 \%$ und 13\%) sowie Diarrhoen (zwischen $10 \%$ und 13\%) sind weniger häufig als Übelkeit, weisen jedoch immer noch deutliche Differenzen zu den Vergleichsgruppen auf (Erbrechen jeweils 2\% der Patienten, Diarrhoen jeweils 5\%).

Die wichtigste Maßnahme zur Vermeidung von Übelkeit und Erbrechen ist das langsame Auftitrieren der Dosis des GLP-1-RA. Außerdem ist die Aufklärung des Patienten zum überwiegend vorübergehenden Charakter der gastrointestinalen Nebenwirkungen wichtig, um Therapieabbrüche zu vermeiden (Bawa 2013, Li 2012). Der positive Effekt einer langsamen Auftitrierung zur Reduktion gastrointestinaler Nebenwirkungen konnte insbesondere in Studien gezeigt werden, in denen Liraglutid als Komponente einer Fixkombinationstherapie zusammen mit Insulin degludec (IDegLira, Xultophy $^{\circledR}$, Novo Nordisk A/S, Dänemark) bei Patienten mit Typ-2Diabetes untersucht wurde. Durch die in diesen Studien (DUAL I, DUAL II) praktizierte langsame Auftitrierung über mehrere Wochen gelang es, den Anteil der von Übelkeit betroffenen Patienten auf unter $10 \%$ zu senken (Buse 2014, Gough 2014) ( $\bullet$ Abb. 1b).

\section{Akute Pankreatitis \\ $\nabla$}

Analysen von Datenbanken zu unerwünschten Ereignissen ergaben Hinweise für ein erhöhtes Risiko von akuten Pankreatitisereignissen unter einer Inkretin-basierten Therapie (Faillie 2014). Nachfolgende Untersuchungen konnten diesen Verdacht jedoch nicht bestätigen. So ergab eine populationsbasierte Kohortenstudie mit 20748 neu auf eine Inkretin-basierte Therapie eingestellten Patienten mit Typ-2-Diabetes eine Ereignisrate von 1,45 pro 1000 Patientenjahre (95\% CI: 0,99-2,11) im Vergleich zu 1,47 (95\% CI: 1,23-1,76) unter Sulfonylharnstoffen (51 712 Patienten) (Faillie 2014). Eine Metaanalyse zu 25 Studien konnte weder

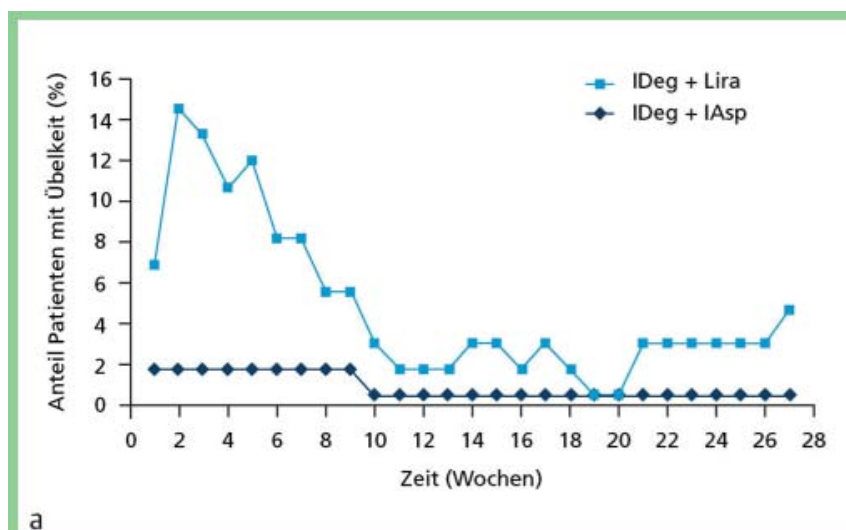

Abb. 1 Anteil an Patienten mit Übelkeit unter Liraglutid bei Intensivierung einer Basalinsulintherapie. a mit Liraglutid: Start mit $0,6 \mathrm{mg} / \mathrm{Tag}$, nach 1. Woche Steigerung auf $1,2 \mathrm{mg} / \mathrm{dl}$, nach 5 . Woche weitere Erhöhung auf $1,8 \mathrm{mg}$ entsprechend dem Nüchternblutzuckerwert (modifiziert nach Mathieu et al. 2014, @ 2014 John Wiley \& Sons Ltd., Abdruck mit Genehmigung), b mit der Fixkombination IDegLira (1 Dosisschritt enthält

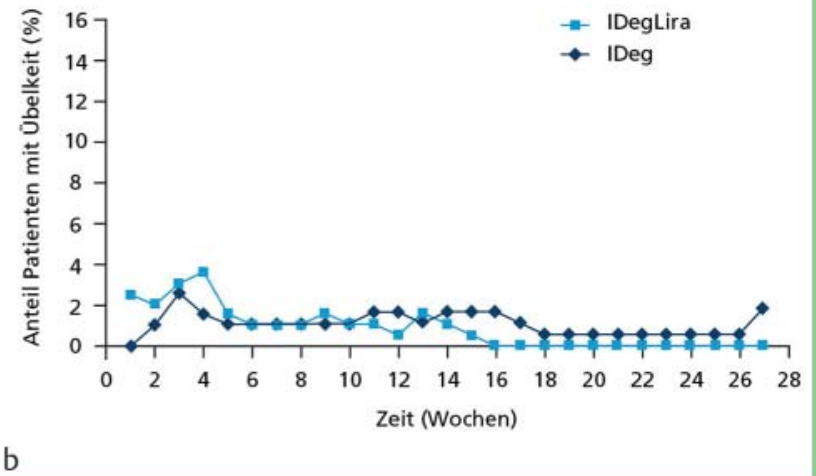

$1 \mathrm{E}$ Insulin degludec und $0,036 \mathrm{mg}$ Liraglutid): Start mit 16 Dosisschritten (0,6 mg Liraglutid, 16 E Insulin degludec), 2-mal wöchentliche Titration mit \pm 2 Dosisschritten, entsprechend dem Nüchternblutzucker, auf durchschnittlich 1,6 mg Liraglutid (45 E Insulin degludec) am Studienende (modifiziert nach Buse et al. 2014, @ American Diabetes Association 2014, Abdruck mit Genehmigung). 
für Exenatid (OR 0,84; 95 \% CI 0,58 - 1,22) noch für Liraglutid (OR 0,97; 95\% CI 0,21-4,39) ein erhöhtes Pankreatitisrisiko zeigen (Alves 2012). Eine weitere Metaanalyse (Stand März 2013) bestätigte dies für die Gruppe der Inkretin-basierten Therapien (Li 2014). Basierend auf 10 Exenatid-Studien lassen sich 3 Ereignisse bei 1968 Patienten (0,15\%) versus 4 Ereignisse bei 2030 Patienten in den Vergleichsgruppen (0,20\%) ermitteln und basierend auf 8 Liraglutid-Studien 4 Ereignisse bei 3643 Patienten $(0,11 \%)$ versus 1 Ereignis bei 1930 Patienten in den Vergleichsgruppen (0,05\%) (Li 2014). Mit diesen Zahlen kann den ursprünglichen Bedenken, dass GLP-1-RA vermehrt akute Pankreatitiden auslösen könnten, begegnet werden.

\section{Exenatid}

Exenatid 2-mal täglich kann gemäß europäischer Zulassung bei Erwachsenen mit Typ-2-Diabetes nicht nur in Kombination mit oralen Antidiabetika, sondern auch mit Basalinsulin angewandt werden. Aufgrund einer mittleren terminalen HWZ von 2,4 Stunden ist eine 2-mal tägliche subkutane Injektion notwendig (Fachinformation Byetta $\left.{ }^{\circledR}\right)$. Die Anfangsdosis von 2-mal täglich $5 \mu \mathrm{g}$ Exenatid sollte zur besseren Verträglichkeit für mindestens einen Monat beibehalten werden, bevor gegebenenfalls auf 2-mal täglich $10 \mu$ g Exenatid gesteigert wird (Fachinformation Byetta ${ }^{\circledR}$ ).

Eine Metaanalyse zu 14 placebokontrollierten Studien weist auf eine Dosisabhängigkeit der mit Exenatid erreichten $\mathrm{HbA}_{1 \mathrm{c}}-\mathrm{Re}-$ duktion hin $(-0,68 \%$ in 5 - $\mu$ g-Gruppen versus $-0,99 \%$ in $10-\mu g$ Gruppen; einschließlich jeweils hiermit bzgl. Dosis äquivalente langwirksame Formulierungen) (Nikfar 2012). Hinsichtlich des Nüchternblutzuckers ergab sich eine mittlere Reduktion um

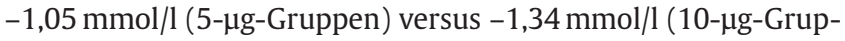
pen). Der Effekt auf das Körpergewicht war mit einer mittleren Reduktion von $-0,56 \mathrm{~kg}$ in den 5 - $\mu$ g-Gruppen versus $-1,24 \mathrm{~kg}$ in den 10- $\mu$ g-Gruppen unterschiedlich stark ausgeprägt.

Die 4B-Studie von Diamant et al. (2014) ergab für die Therapieeskalation einer Basalinsulintherapie mit Exenatid im Vergleich zu einer intensivierten konventionellen Therapie (ICT) bei vergleichbarer $\mathrm{HbA}_{1 \mathrm{c}}$-Reduktion signifikante Verbesserungen hinsichtlich nicht-nächtlicher Hypoglykämien, des Körpergewichts und der Therapiezufriedenheit.

\section{Liraglutid}

Liraglutid ist ebenfalls arzneimittelrechtlich für Erwachsene mit Typ-2-Diabetes in Kombination mit oralen Antidiabetika und/ oder Basalinsulin zugelassen. Die 1-mal tägliche Liraglutid-Anfangsdosis beträgt $0,6 \mathrm{mg}$. Frühestens nach einer Woche kann auf 1,2 mg Liraglutid erhöht werden, wobei ein Teil der Patienten im Verlauf von einer weiteren Steigerung der 1-mal täglichen Liraglutid-Injektion auf $1,8 \mathrm{mg}$ profitieren kann (Fachinformation Victoza $^{\circledR}$ ). Liraglutid ist ein GLP-1-RA mit längerer HWZ von ca. 13 Stunden (Agersø 2002, Elbrønd 2002). Liraglutid-Maximalkonzentrationen werden 8-12 Stunden nach subkutaner Injektion erreicht. Die Liraglutid-Exposition steigt proportional zur Dosis (Agersø 2002). Es konnte kein Organ identifiziert werden, in dem die Hauptelimination stattfindet. Es wird davon ausgegangen, dass Liraglutid ähnlich wie andere Peptide metabolisiert wird (Helleberg H 2008).

Gemäß einer Metaanalyse zu den sechs LEAD-Studien („Liraglutide Effect and Action in Diabetes“) und einer weiteren Studie, die Liraglutid mit Sitagliptin verglich, erreichten durchschnittlich $40 \%$ der mit Liraglutid 1,8 mg behandelten Patienten mit Typ-2-
Diabetes einen $\mathrm{HbA}_{1 \mathrm{c}}$-Wert $<7,0 \%$, ohne dass es zu einer Hypoglykämie oder einer Gewichtszunahme kam (Zinman 2012). Unter Liraglutid 1,2 mg war die $\mathrm{HbA}_{1 \mathrm{c}}$-Reduktion geringer, so dass mit $32 \%$ der Patienten dieser kombinierte Endpunkt signifikant seltener eintrat. Diese Rate war außerdem geringer unter Exenatid (25\%), Insulin glargin (15\% in LEAD 5), Sulfonylharnstoff ( $8 \%$ in LEAD 2 und LEAD 3), Thiazolidinedion (6\% in LEAD 4) und Sitagliptin (11\% (Pratley2010)). Die im Vergleich zu Sitagliptin durchgeführte 26-wöchige Studie ergab zudem Hinweise auf eine Abhängigkeit von der Liraglutid-Dosis hinsichtlich der mittleren Reduktionen des Nüchternblutzuckers $(-2,14 \mathrm{mmol} / \mathrm{l}$ unter $1,8 \mathrm{mg}$ Liraglutid versus $-1,87 \mathrm{mmol} / \mathrm{l}$ unter $1,2 \mathrm{mg}$ Liraglutid versus $-0,83 \mathrm{mmol} / \mathrm{l}$ unter Sitagliptin) (Pratley 2010). Das Körpergewicht verringerte sich um durchschnittlich $-3,38 \mathrm{~kg}$ unter $1,8 \mathrm{mg}$ Liraglutid versus $-2,86 \mathrm{~kg}$ unter $1,2 \mathrm{mg}$ Liraglutid und $-0,96 \mathrm{~kg}$ unter Sitagliptin (Pratley 2010).

Im September 2014 wurde mit Xultophy ${ }^{\circledR}$ eine Fixkombination aus Liraglutid und Insulin degludec (IDeg) zugelassen. Beide Wirkstoffe werden jeweils 1-mal täglich angewendet, so dass diese beiden Substanzen für eine Fixkombination geeignet waren (Kumar 2014).

\section{Albiglutid}

Albiglutid hat im März 2014 die arzneimittelrechtliche Zulassung von der Europäischen Arzneimittel-Agentur (EMA) bei erwachsenen Patienten mit Typ-2-Diabetes sowohl zur Monotherapie bei Metformin-Kontraindikation oder -Unverträglichkeit als auch zur Kombinationstherapie mit anderen Antidiabetika einschließlich Basalinsulin erhalten (Fachinformation Eperzan ${ }^{\circledR}$ ). Die HWZ ist mit 5 Tagen deutlich länger als bei Exenatid und Liraglutid, so dass eine 1-mal wöchentliche subkutane Injektion von $30 \mathrm{mg} \mathrm{Al-}$ biglutid mit eventueller Steigerung auf $50 \mathrm{mg}$ ausreicht.

\section{Intensivierung einer Basalinsulintherapie mit GLP-1-RA $\nabla$}

\section{Rationale}

Die Entwicklung der GLP-1-RA fokussierte sich zunächst auf die Kombinationstherapie mit oralen Antidiabetika bei Typ-2-Diabetes. Im Behandlungsalltag zeigt sich jedoch, dass GLP-1-RA häufig mit Insulinen kombiniert werden. So verwendeten $38 \%$ bzw. $43 \%$ der in Dänemark mit Liraglutid oder Exenatid behandelten Patienten zugleich Insulin (Pottegard 2014). Basalinsulin und GLP1-RA ergänzen sich hinsichtlich des Wirkmechanismus und des Nebenwirkungsprofils ( $\bullet$ Tab. 1 ). Die häufig bei der Einleitung oder Intensivierung einer Insulintherapie geäußerten Bedenken hinsichtlich Hypoglykämien und Gewichtszunahme (Holst 2013) bestehen bei GLP-1-RA nicht.

Basalinsuline, die mit einem GLP-1-RA kombiniert werden können, sind NPH-Insulin, Insulin glargin (IGlar), Insulin detemir (IDet) und das im Jahre 2013 zugelassene Insulin degludec (IDeg). Nach Zugabe eines GLP-1-RA zu einer laufenden Basalinsulintherapie sollte die Reduktion der Insulindosis in Betracht gezogen werden, um Hypoglykämien zu vermeiden (Fachinformationen Victoza $^{\circledR}$, Fachinformation Byetta ${ }^{\circledR}$, Fachinformation Eperzan ${ }^{\circledR}$ ).

\section{Studien zur Intensivierung einer Insulintherapie mit Exenatid oder Liraglutid}

Nach einer „Proof-of-Concept“-Studie zur Kombination von Exenatid mit Insulin glargin (IGlar) (Arnolds 2010) folgte die erste größere randomisierte placebokontrollierte Studie zur Kombination von Exenatid mit Insulin glargin (Buse 2011) ( Tab.2). Bei 
Tab. 1 Komplementäre Eigenschaften von Basalinsulin und GLP-1-RA (modifiziert und ergänzt nach Balena et al. (2013) und Jendle et al. (2012)).

\begin{tabular}{|c|c|c|}
\hline Eigenschaft & Basalinsulin & GLP-1-RA \\
\hline $\begin{array}{l}\text { Wirkmecha- } \\
\text { nismus }\end{array}$ & $\begin{array}{l}\text { - systemische Insulin- } \\
\text { konzentration } \uparrow \text { (auch } \\
\text { endogenes Insulin) } \\
\text { - Glucagonsekretion } \downarrow \\
\text { - hepatische Glucose- } \\
\text { produktion } \downarrow\end{array}$ & $\begin{array}{l}\text { - Stimulation der glucose- } \\
\text { abhängigen Insulin- } \\
\text { sekretion } \\
\text { - postprandiale Glucagon- } \\
\text { sekretion } \downarrow \\
\text { - hepatische Glucose- } \\
\text { produktion } \downarrow \\
\text { - Magenentleerung } \\
\text { verzögert } \\
\text { - Sättigungsgefühl } \uparrow \\
\text { - Glucoseresorption } \downarrow\end{array}$ \\
\hline $\begin{array}{l}\text { führende } \\
\text { Wirkungen }\end{array}$ & $\begin{array}{l}\text { - Nüchternblutzucker } \downarrow \\
\text { - Blutzucker zwischen den } \\
\text { Mahlzeiten } \downarrow\end{array}$ & $\begin{array}{l}\text { - postprandialer Blut- } \\
\text { zucker } \downarrow \\
\text { - Nüchternblutzucker } \downarrow \\
\text { insbesondere bei län- } \\
\text { gerer Halbwertszeit } \\
\text { - Körpergewicht } \downarrow\end{array}$ \\
\hline $\begin{array}{l}\text { führende } \\
\text { Nebenwir- } \\
\text { kungen }\end{array}$ & $\begin{array}{l}\text { - Hypoglykämien } \\
\text { - Körpergewicht } \uparrow\end{array}$ & $\begin{array}{l}\text { - Übelkeit, Erbrechen, } \\
\text { Diarrhoe }\end{array}$ \\
\hline
\end{tabular}

Patienten mit einem Ausgangs- $\mathrm{HbA}_{1 \mathrm{c}}$ von $\leq 8 \%$ wurde zu Beginn der Therapie mit Exenatid die IGlar-Dosis um 20\% reduziert. Nach Woche 5 wurde bei allen Patienten die IGlar-Dosis angepasst, um einen Nüchternblutzucker von $<5,6 \mathrm{mmol} / \mathrm{l}$ ( $<100 \mathrm{mg} /$ dl) zu erreichen („Treat-to-Target“-Design). Dies führte zu einer Steigerung der IGlar-Dosis bis zum Studienende um durchschnittlich 13 Einheiten/Tag im Vergleich zu 20 Einheiten/Tag in der Placebogruppe. Aufgrund des „Treat-to-Target“-Designs war kein Unterschied zwischen den Gruppen hinsichtlich der Reduktion des Nüchternblutzuckers zu erwarten, jedoch waren unter Exenatid der $\mathrm{HbA}_{1 \mathrm{c}}$-Wert sowie der postprandiale Blutzucker nach dem Frühstück und nach dem Abendessen signifikant reduziert, ohne dass es zu einem Anstieg bei den Hypoglykämien kam ( $\bullet$ Tab.2). Außerdem war der Gewichtsverlauf unter Exenatid signifikant verbessert ( $\bullet$ Tab. 2).

Die erste randomisierte, kontrollierte Studie zur Kombination von Liraglutid mit Basalinsulin schloss 84 Patienten mit Typ2-Diabetes und einer Insulindosis von mindestens $10 \mathrm{E} / \mathrm{Tag}$ ein (Li 2012). Als Zielwerte für den Blutzuckerspiegel wurden $\leq 6,1 \mathrm{mmol} / \mathrm{l}$ nüchtern und $\leq 8 \mathrm{mmol} / 12$ Stunden postprandial definiert. Hierzu konnten Liraglutid, Insulin oder die oralen Antidiabetika individuell optimiert werden. Nach 12 Wochen zeigten

Tab.2 Wirksamkeit von Exenatid in randomisierten kontrollierten Studien zur Intensivierung einer bestehenden Basalinsulintherapie bei Typ-2-Diabetes.

\begin{tabular}{|c|c|c|c|c|c|c|c|c|c|}
\hline \multirow[t]{2}{*}{ Referenz } & \multirow[t]{2}{*}{ Vortherapie } & \multirow[t]{2}{*}{ Studienarme } & \multirow{2}{*}{$\begin{array}{l}\mathrm{HbA}_{1 c^{-}} \\
\text {Verlauf } \\
(\%)\end{array}$} & \multirow{2}{*}{$\begin{array}{l}\mathrm{HbA}_{1 \mathrm{c}} \leq 7 \% \\
\text { (\%-Anteil } \\
\text { Pat.) }\end{array}$} & \multirow{2}{*}{$\begin{array}{l}\text { Nü-BZ } \\
(\mathrm{mmol} / \mathrm{l})\end{array}$} & \multirow{2}{*}{$\begin{array}{l}\text { BZ post- } \\
\text { prandial } \\
\text { (mmol/l) }\end{array}$} & \multirow{2}{*}{$\begin{array}{l}\text { Gewicht } \\
\text { (kg) }\end{array}$} & \multicolumn{2}{|c|}{ Hypoglykämien } \\
\hline & & & & & & & & $\begin{array}{l}\text { schwer } \\
\text { (Pat. mit } \\
\text { Ereignis) }\end{array}$ & leicht \\
\hline \multirow{3}{*}{$\begin{array}{l}\text { Arnolds } \\
2010 \\
\text { 4-wöchige } \\
\text { offene RCT } \\
\text { (Treat-to- } \\
\text { Target) } \\
\mathrm{n}=48\end{array}$} & \multirow[t]{3}{*}{$\begin{array}{l}\text { Met } \pm \text { SH oder } \\
\text { Basalinsulin } \pm \\
\text { Met }\end{array}$} & $\begin{array}{l}\text { Exenatid + } \\
\text { IGlar + Met }\end{array}$ & $\begin{array}{l}-1,8 \% \\
(p=0,0154 \\
\text { vs. IGlar } \\
+ \text { Met) }\end{array}$ & $80 \%$ & $\begin{array}{l}5,2 \rightarrow 4,6 \\
(p=0,0018)\end{array}$ & $\begin{array}{l}-17 \% \\
(p=0,0036 \text { vs. } \\
\text { IGlar })+ \text { Met }\end{array}$ & $\begin{array}{l}-0,9 \mathrm{~kg} \\
(p=0,038 \\
\text { vs. IGlar) } \\
+ \text { Met }\end{array}$ & $\mathrm{n}=0$ & $\begin{array}{l}\text { Ereignisse/Pat.-jahr } \\
\text { gesamt: } 1,7 \\
\text { nächtlich: } 0\end{array}$ \\
\hline & & $\begin{array}{l}\text { Sita + IGlar + } \\
\text { Met }\end{array}$ & $-1,5 \%$ & $88 \%$ & $\begin{array}{l}5,3 \rightarrow 4,7 \\
(p=0,0016)\end{array}$ & $\begin{array}{l}-20 \% \\
(p=0,0008 \text { vs. } \\
\text { IGlar })+ \text { Met }\end{array}$ & $+0,1 \mathrm{~kg}$ & $\mathrm{n}=0$ & $\begin{array}{l}\text { Ereignisse/Pat.-jahr } \\
\text { gesamt: } 2,5 \\
\text { nächtlich: } 0\end{array}$ \\
\hline & & IGlar + Met & $-1,2 \%$ & $63 \%$ & $\begin{array}{l}5,2 \rightarrow 4,9 \\
(p=0,21)\end{array}$ & - & $+0,4 \mathrm{~kg}$ & $\mathrm{n}=0$ & $\begin{array}{l}\text { Ereignisse/Pat.-jahr } \\
\text { gesamt: } 1,6 \\
\text { nächtlich: } 0\end{array}$ \\
\hline \multirow{2}{*}{$\begin{array}{l}\text { Buse } 2011 \\
\text { 30-wöchi- } \\
\text { ge doppel- } \\
\text { blinde RCT } \\
\text { (Treat-to- } \\
\text { Target) } \\
\mathrm{n}=259\end{array}$} & \multirow[t]{2}{*}{$\begin{array}{l}\text { IGlar } \pm \\
\text { Met } \\
\text { und/oder } \\
\text { Pioglitazon }\end{array}$} & $\begin{array}{l}\text { Exenatid }+ \\
\text { IGlar } \pm \text { Met } \\
\text { und/oder Pio- } \\
\text { glitazon }\end{array}$ & $-1,74 \%^{1}$ & $60 \%^{2}$ & $-1,6$ & \multirow{2}{*}{$\begin{array}{l}\text { Differenz zw. } \\
\text { Gruppen }{ }^{3} \text { : } \\
\text { morgens: }-1,8 \\
\text { mittags: }-0,3 \\
\text { abends: }-1,7\end{array}$} & $-1,8 \mathrm{~kg}^{4}$ & $\mathrm{n}=0$ & $\begin{array}{l}\text { Anteil Pat. } \\
\text { gesamt: } 24,8 \% \\
\text { nächtlich: } 16,8 \%\end{array}$ \\
\hline & & $\begin{array}{l}\text { Placebo + } \\
\text { IGlar } \pm \text { Met } \\
\text { und/oder Pio- } \\
\text { glitazon }\end{array}$ & $-1,04 \%^{1}$ & $35 \%^{2}$ & $-1,5$ & & $+1,0 \mathrm{~kg}^{4}$ & $\mathrm{n}=1$ & $\begin{array}{l}\text { Anteil Pat. } \\
\text { gesamt: } 28,7 \% \\
\text { nächtlich: } 26,2 \%\end{array}$ \\
\hline \multirow{2}{*}{$\begin{array}{l}\text { Diamant } \\
2014 \\
30 \text {-wöchi- } \\
\text { ge offene } \\
\text { RCT (Treat- } \\
\text { to-Target) } \\
\mathrm{n}=627\end{array}$} & \multirow[t]{2}{*}{$\begin{array}{l}\text { IGlar }+ \\
\text { Met } \pm \text { SH }\end{array}$} & $\begin{array}{l}\text { Exenatid + } \\
\text { IGlar }+ \\
\text { Met }\end{array}$ & $-1,13 \%$ & $49,6 \%$ & $-0,46^{5}$ & \multirow{2}{*}{$\begin{array}{l}\text { morgens: } \\
-2,57 \text { vs. } \\
-2,30 \\
\text { mittags: } \\
-2,18 \text { vs. } \\
-3,11^{6} \\
\text { abends: } \\
-2,88 \text { vs. } \\
-3,16\end{array}$} & $-2,5 \mathrm{~kg}^{7}$ & $\mathrm{n}=2$ & $\begin{array}{l}\text { Anteil Pat. } \\
\text { gesamt: } 30 \% \\
\text { nächtlich: } 25 \%\end{array}$ \\
\hline & & $\begin{array}{l}\text { Insulin lispro + } \\
\text { IGlar + Met }\end{array}$ & $-1,10 \%$ & $49,0 \%$ & $+0,18^{5}$ & & $+2,1 \mathrm{~kg}^{7}$ & $\mathrm{n}=7$ & $\begin{array}{l}\text { Anteil Pat. } \\
\text { gesamt: } 41 \% \\
\text { nächtlich: } 27 \%\end{array}$ \\
\hline
\end{tabular}

IGlar: Insulin glargin; Sita: Sitagliptin; RCT: randomisierte kontrollierte Studie, SH: Sulfonylharnstoff, Met: Metformin; Ereignisse/Pat.-jahr: Ereignisse/Patientenjahr.

${ }^{1}$ Differenz zwischen Gruppen: $-0,69 \%(95 \% \mathrm{Cl}-0,93$ bis $-0,46 \%)$; $<0,001$

2 Differenz zwischen Gruppen $25 \%$ ( $95 \%$ Cl $12 \%$ bis $39 \%)$; $p<0,001$.

${ }^{3}$ Differenzen zwischen Gruppen signifikant nach Frühstück ( $95 \% \mathrm{Cl}-2,5$ bis $-1,2$ mmol/l; $\left.\mathrm{p}<0,001\right)$ und Abendessen $(95 \% \mathrm{Cl}-2,3$ bis $-1,1$, mmol/l; $\mathrm{p}<0,001)$, nicht jedoch nach dem Mittagessen $(p=0,32)$

${ }^{4}$ Differenz zwischen Gruppen: $-2,7 \mathrm{~kg}(95 \% \mathrm{Cl}:-3,7$ bis $-1,7)$; $\mathrm{p}<0,001$.

${ }^{5}$ Mittlere Differenz: $-0,64 \mathrm{mmol} / \mathrm{l}(95 \% \mathrm{Cl}:-1,05$ bis $-0,24) ; \mathrm{p}=0,002$.

${ }^{6}$ Mittlere Differenz: + 0,93 mmol (95\% Cl: 0,52 bis 1,34); $\mathrm{p}<0,001$.

${ }^{7}$ Mittlere Differenz: $-4,6 \mathrm{~kg}$ (95\% Cl: $-5,2$ bis $\left.-3,9\right) ; \mathrm{p}<0,001$. 
sich für die Liraglutid-Gruppe im Vergleich zum anderen Studienarm signifikant verbesserte postprandiale Blutzuckerwerte und Gewichtsverläufe, während Reduktionen von Nüchternblutzucker und $\mathrm{HbA}_{1 \mathrm{c}}$ vergleichbar waren ( $\bullet$ Tab.3). Die mittlere Insulindosis wurde in der Liraglutid-Gruppe bis Woche 12 um $66 \%$ reduziert, wobei $38 \%$ der Patienten Insulin absetzen konnten. Dagegen war in der Insulintitrationsgruppe ein Anstieg der Insulindosis um $28 \%$ zu verzeichnen. Außerdem war in der Liraglutid-Gruppe häufiger ein Absetzen von Sulfonylharnstoffen (85,7\% versus 22,2\%; $p<0,05$ ), Gliniden ( $87,5 \%$ versus $46,2 \%$; $\mathrm{p}<0,05)$ und $\alpha$-Glukosidaseinhibitoren (66,7\% versus $23,1 \%$; $\mathrm{p}<0,05)$ möglich. Leichte Hypoglykämien waren in der Liraglutid-Gruppe signifikant seltener (11,9\% versus $31,0 \%$; $=0,033)$ (o Tab. 3).

Zwei randomisierte, kontrollierte Studien konnten trotz einer relativ kleinen Fallzahl ( $\mathrm{n}=50$ bzw. $\mathrm{n}=37$ ) eine signifikant stärkere $\mathrm{HbA}_{1 \mathrm{c}}$-Reduktion für Liraglutid 1,2 oder $1,8 \mathrm{mg}$ im Vergleich zu einer Insulintitration zeigen (De Wit 2014, Lane 2014) ( Tab.3). Beide Studien legten den Fokus auf das Körpergewicht, da die Patienten bei Studieneinschluss entweder adipös (Lane 2014) oder durch Gewichtszunahme $\geq 4 \mathrm{~kg}$ seit Start der Insulintherapie auffällig sein mussten (de Wit 2014). In beiden Studien waren verschiedene Arten der Insulintherapie zulässig, einschließlich kurz wirksamer Insuline. Nach 26 Wochen bzw. 6 Monaten war der Verlauf des Körpergewichts in den Liraglutid-Gruppen um jeweils ca. $5 \mathrm{~kg}$ günstiger als ohne Liraglutid ( $\bullet$ Tab.3) (de Wit 2014, Li 2014). Die Insulindosis konnte jeweils deutlich reduziert und die Insulintherapie in der Studie von de Wit et al. (2014) bei 5 von 26 Patienten (19\%) abgesetzt werden.

Die Ergebnisse einer größeren randomisierten, placebokontrollierten Studie zur Intensivierung einer Basalinsulintherapie mit Liraglutid liegen bisher nur als Kongressabstract vor (Lahtela 2014). In der Vergleichsgruppe war eine Erhöhung der Insulindosis nicht zulässig, so dass die Ergebnisse den isolierten Effekt der Zugabe von Liraglutid zu einer Basalinsulintherapie repräsentieren. Nach 26 Wochen waren $\mathrm{HbA}_{1 \mathrm{c}}$, Nüchternblutzucker, postprandialer Blutzucker und Körpergewicht signifikant verbessert, ohne dass es zu einer schweren Hypoglykämie bei den 226 mit Liraglutid behandelten Patienten kam ( $\bullet$ Tab.3).

\section{Direkter Vergleich von GLP-1-RA mit kurz wirksamem Insulin}

Bisher liegen Ergebnisse aus drei randomisierten, kontrollierten, offenen Studien zum direkten Vergleich von Exenatid, Liraglutid oder Albiglutid mit kurz wirksamen Insulinanaloga vor (Diamant 2014, Mathieu 2014, Rosenstock 2014). Während in den Studien zu Exenatid (4B-Study) und Albiglutid (HARMONY 6) das kurz wirksame Insulin (Insulin lispro) sofort zu allen drei Hauptmahlzeiten appliziert wurde (Diamant 2014, Rosenstock 2014), verfolgte die Liraglutid-Studie (BEGIN VICTOZA ADD-ON) in der Vergleichsgruppe eine Basal-Plus-Strategie, bei der das kurz wirksame Insulin (Insulin aspart) zunächst nur zu einer Mahlzeit gegeben wird, mit Option zur Dosissteigerung im weiteren Verlauf (Mathieu 2014, Owens 2013). Gemäß FullSTEP-Studie ist ein schrittweises Vorgehen bzgl. Bolusinsulin im Vergleich zur sofortigen Bolusinsulingabe zu jeder Mahlzeit mit einem geringeren Hypoglykämierisiko assoziiert bei Nichtunterlegenheit bzgl. $\mathrm{HbA}_{1 \mathrm{c}}$-Reduktion am Studienende nach 32 Wochen (Rodbard 2013a). In den mit Exenatid und Albiglutid durchgeführten Studien erhielten alle Patienten das Basalinsulin Insulin glargin, während die als Anschlussstudie zur BEGIN-ONCE-LONG-Exten- sionsstudie (Rodbard 2013b) konzipierte BEGIN-VICTOZA-ADDON-Studie IDeg verwendete.

Eine signifikant stärkere Reduktion des $\mathrm{HbA}_{1 \mathrm{c}}$ im Vergleich zum kurz wirksamen Insulinanalogon wurde nur in der LiraglutidStudie erreicht, in der das kurz wirksame Insulin allerdings nur zu einer Mahlzeit verabreicht wurde (Mathieu 2014). Dagegen ergab die Exenatid-Studie hinsichtlich des Nüchternblutzuckers einen Vorteil für den GLP-1-RA (Diamant 2014). Möglicherweise war ein solcher Effekt in der Liraglutid-Studie nicht nachweisbar, da bereits IDeg im Vergleich zu Insulin glargin gemäß zwei von drei Studien zu insulinnaivem Typ-2-Diabetes eine signifikant stärkere Reduktion des Nüchternblutzuckers um $0,4 \mathrm{mmol} / \mathrm{l}$ bewirken kann (Merker 2013). Außerdem orientierte sich die Dosistitration für Liraglutid und IDeg in der Liraglutid-Studie durch das Treat-to-Target-Design am Nüchternblutzucker.

In allen drei Studien zeichneten sich Vorteile für die beiden GLP1-RA hinsichtlich Hypoglykämien ab, wobei die Liraglutid-Studie eine Reduktion um $87 \%$ von 8,15 auf 1,00 Ereignisse pro Patientenjahr ergab $(p<0,0001)$ und in der Albiglutid-Studie eine Reduktion von 3,09 auf 1,31 Ereignisse pro Patientenjahr dokumentiert wurde (kein p-Wert angegeben). Erneut bestätigte sich der positive Einfluss der GLP-1-RA auf das Körpergewicht mit Behandlungsdifferenzen im Vergleich zur Intensivierung mit kurz wirksamen Insulinanaloga von ca. 4 - $5 \mathrm{~kg}$ für Exenatid ( $\bullet$ Abb. 2) und Liraglutid (Diamant 2014, Mathieu 2014) und 1,5 kg für Albiglutid (Rosenstock 2014). Eine Verbesserung hinsichtlich Fettstoffwechselparametern konnte weder für die GLP-1-RA noch für die beiden kurz wirksamen Insulinanaloga gezeigt werden (Diamant 2014, Mathieu 2014).

\section{Studie zur Fixkombination IDegLira}

Eine randomisierte, kontrollierte Doppelblindstudie verglich die Fixkombination aus Insulin degludec und Liraglutid (IDegLira) mit Insulin degludec (IDeg) bei Patienten mit Typ-2-Diabetes, die zugleich Metformin erhielten (Buse 2014). Eventuell zuvor verabreichte Sulfonylharnstoffe oder Glinide wurden zum Zeitpunkt der Randomisierung abgesetzt. Dies traf auf 52\% bzw. 51 \% der Pa-

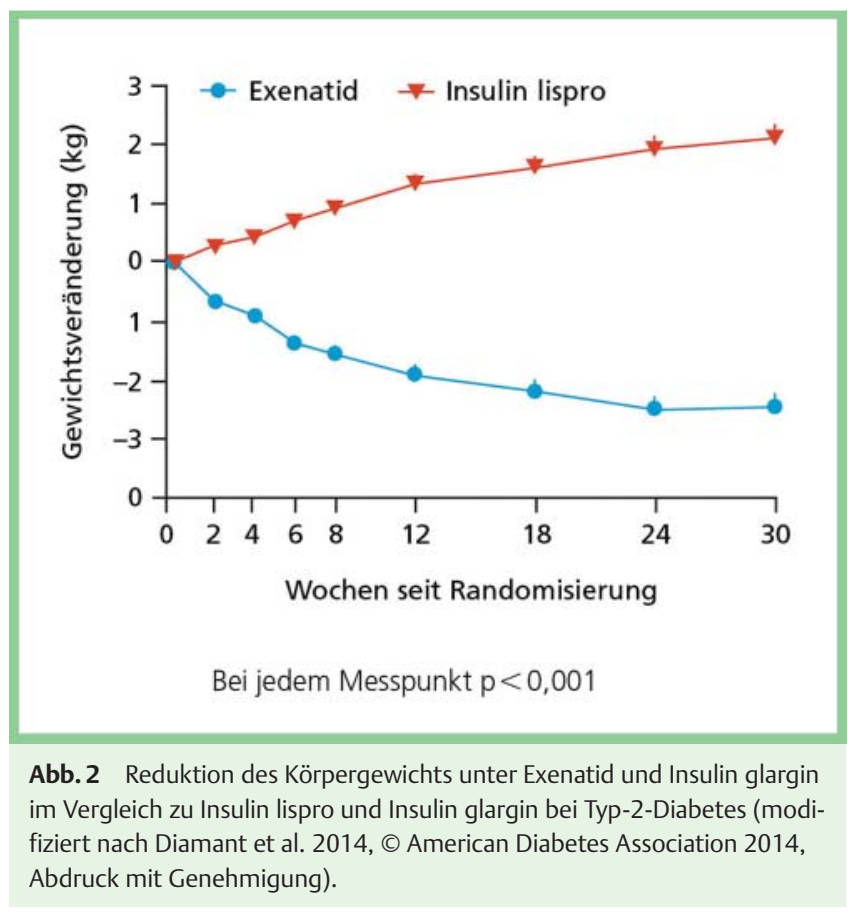


Tab. 3 Wirksamkeit von Liraglutid in randomisierten kontrollierten Studien zur Intensivierung einer bestehenden Insulintherapie bei Typ-2-Diabetes.

\begin{tabular}{|c|c|c|c|c|c|c|c|c|c|}
\hline \multirow[t]{2}{*}{ Referenz } & \multirow[t]{2}{*}{ Vortherapie } & \multirow[t]{2}{*}{ Studienarme } & \multirow{2}{*}{$\begin{array}{l}\mathrm{HbA}_{1 c^{-}} \\
\text {Verlauf } \\
(\%)\end{array}$} & \multirow{2}{*}{$\begin{array}{l}\mathrm{HbA}_{1 \mathrm{c}} \leq 7,0 \% \\
\text { (\%-Anteil } \\
\text { Pat.) }\end{array}$} & \multirow{2}{*}{$\begin{array}{l}\text { Nü-BZ } \\
(\mathrm{mmol} / \mathrm{l})\end{array}$} & \multirow{2}{*}{$\begin{array}{l}\text { BZ postpran- } \\
\text { dial (mmol/l) }\end{array}$} & \multirow{2}{*}{$\begin{array}{l}\text { Gewicht } \\
\text { (kg) }\end{array}$} & \multicolumn{2}{|c|}{ Hypoglykämien } \\
\hline & & & & & & & & $\begin{array}{l}\text { schwer } \\
\text { (Pat. mit } \\
\text { Ereignis) }\end{array}$ & leicht \\
\hline \multirow[t]{2}{*}{$\begin{array}{l}\text { Li } 2012 \\
\text { 12-wöchige } \\
\text { offene RCT } \\
n=84\end{array}$} & \multirow{2}{*}{$\begin{array}{l}\text { Insulin } \geq 10 \mathrm{E} / \\
\text { Tag } \\
\text { (Glar, NPH-In- } \\
\text { sulin oder } \\
\text { Misch-insulin) } \\
\pm \text { OAD }\end{array}$} & $\begin{array}{l}\text { Insulin }+ \\
\text { Liraglutid } \pm \\
\text { OAD }\end{array}$ & $-1,9 \%$ & $73,8 \%$ & $-1,56$ & $-5,40^{1}$ & $-5,6 \mathrm{~kg}^{2}$ & $\mathrm{n}=0$ & $\begin{array}{l}\text { Anteil Pat. } \\
11,9 \% \\
\text { ( } p=0,033 \text { vs. } \\
\text { Vergl.gruppe) }\end{array}$ \\
\hline & & $\begin{array}{l}\text { Insulintitration } \\
\pm O A D\end{array}$ & $-1,77 \%$ & $73,8 \%$ & $-1,69$ & $-4,54^{1}$ & $+2,0 \mathrm{~kg}^{2}$ & $n=2$ & $\begin{array}{l}\text { Anteil Pat. } \\
31,0 \%\end{array}$ \\
\hline \multirow{2}{*}{$\begin{array}{l}\text { De Wit } 2014 \\
\text { 26-wöchige } \\
\text { offene RCT } \\
\text { (Treat-to- } \\
\text { Target) } \\
\mathrm{n}=50\end{array}$} & \multirow[t]{2}{*}{$\begin{array}{l}\text { Insulin } \pm \text { Met } \\
\pm \mathrm{SH}\end{array}$} & $\begin{array}{l}\text { Insulin }+ \\
\text { Liraglutid } \pm \\
\text { Met } \pm \text { SH }\end{array}$ & $-0,77 \%^{3}$ & $\begin{array}{l}73 \% \\
\text { ( } p=0,004 \text { vs. } \\
\text { Vergl.gruppe) }\end{array}$ & k. A. & k. A. & $-4,5 \mathrm{~kg}^{4}$ & $\mathrm{n}=0$ & $\begin{array}{l}\text { Ereignisse/Pat.- } \\
\text { jahr } \\
3,85\end{array}$ \\
\hline & & $\begin{array}{l}\text { Insulintitration } \\
\pm \text { Met } \pm \text { SH }\end{array}$ & $+0,01 \%^{3}$ & $29 \%$ & k. A. & k. A. & $+0,9 \mathrm{~kg}^{4}$ & $\mathrm{n}=0$ & $\begin{array}{l}\text { Ereignisse/Pat.- } \\
\text { jahr } \\
2,70\end{array}$ \\
\hline \multirow[t]{2}{*}{$\begin{array}{l}\text { Lane } 2014 \\
6 \text {-monatige } \\
\text { offene RCT } \\
n=37\end{array}$} & \multirow[t]{2}{*}{$\begin{array}{l}\text { Insulin } \geq 100 \\
\text { E/Tag } \pm \text { Met }\end{array}$} & $\begin{array}{l}\text { Insulin }+ \\
\text { Liraglutid } \pm \\
\text { Met }\end{array}$ & $\begin{array}{l}-0,65 \% \\
\text { auf } 7,15 \% \\
\text { (Monat 6; } \\
p=0,047 \\
\text { vs. Vergl. } \\
\text { gruppe) }\end{array}$ & $43 \%$ & \multicolumn{2}{|c|}{$\begin{array}{l}\text { Kontinuierliche Glukose- } \\
\text { messung: Anteil Zeit } \\
>180 \mathrm{mg} / \mathrm{dl} \text { : } \\
\text { Reduktion von } 38 \% \text { auf } 22 \% \\
\text { ( } \mathrm{p} \leq 0,0001 \text { vs. Baseline) }\end{array}$} & $-5,3 \mathrm{~kg}$ & $\mathrm{n}=0$ & k. A. \\
\hline & & $\begin{array}{l}\text { Insulintitration } \pm \\
\text { Met }\end{array}$ & $\begin{array}{l}-0,39 \% \\
\text { auf } 7,40 \% \\
\text { (Monat } 6 \text { ) }\end{array}$ & $31 \%$ & \multicolumn{2}{|c|}{$\begin{array}{l}\text { Anteil Zeit } \\
>180 \mathrm{mg} / \mathrm{dl} \text { : } \\
\text { keine Reduktion }\end{array}$} & $+0,4 \mathrm{~kg}$ & $n=0$ & k. A. \\
\hline \multirow{2}{*}{$\begin{array}{l}\text { Lahtela } \\
2014 \\
\text { 26-wöchige } \\
\text { doppelblin- } \\
\text { de RCT } \\
\mathrm{n}=451\end{array}$} & \multirow[t]{2}{*}{$\begin{array}{l}\text { Insulin } \geq 20 \mathrm{E} / \\
\text { Tag } \pm \text { Met }\end{array}$} & $\begin{array}{l}\text { Insulin + Liraglu- } \\
\text { tid } \pm \text { Met }\end{array}$ & $-1,30 \%^{5}$ & $59,2 \%^{6}$ & $-1,44^{7}$ & $-0,94^{8}$ & $-3,5 \mathrm{~kg}^{9}$ & $\mathrm{n}=0$ & $\begin{array}{l}\text { Anteil Pat. } \\
18,2 \%\end{array}$ \\
\hline & & $\begin{array}{l}\text { Insulin + Placebo } \\
\pm \text { Met }\end{array}$ & $-0,11 \%^{5}$ & $14,0 \%^{6}$ & $-0,16^{7}$ & $-0,37^{8}$ & $-0,4 k g^{9}$ & $\mathrm{n}=0$ & $\begin{array}{l}\text { Anteil Pat. } \\
12,4 \%\end{array}$ \\
\hline \multirow{2}{*}{$\begin{array}{l}\text { Buse } 2014 \\
\text { 26-wöchige } \\
\text { doppelblin- } \\
\text { de RCT } \\
\text { (Treat-to- } \\
\text { Target) } \\
n=298\end{array}$} & \multirow[t]{2}{*}{$\begin{array}{l}\text { Basalinsulin + } \\
\text { Met } \pm \text { SH/Gli- } \\
\text { nide }\end{array}$} & $\begin{array}{l}\text { IDegLira Start: } 16 \\
\text { E/0,6 mg; Titrati- } \\
\text { on auf max. } 50 \mathrm{E} / \\
1,8 \mathrm{mg} \\
+ \text { Met }\end{array}$ & $-1,9 \% 10$ & $\begin{array}{l}60 \% \\
p<0,0001 \text { vs. } \\
\text { Vergl.gruppe) }\end{array}$ & $-3,5^{11}$ & $\begin{array}{l}2,2 \\
\text { Anstieg vs. } \\
\text { vor den } \\
\text { Mahlzeiten }^{12}\end{array}$ & $-2,7 \mathrm{~kg}^{13}$ & $n=1$ & $\begin{array}{l}\text { Anteil Pat. } \\
24 \%\end{array}$ \\
\hline & & $\begin{array}{l}\text { IDeg } \\
\text { Start: } 16 \text { E; Titra- } \\
\text { tion auf max. } 50 \mathrm{E} \\
+ \text { Met }\end{array}$ & $-0,9 \% 10$ & $23 \%$ & $-2,6^{11}$ & $\begin{array}{l}2,4 \\
\text { Anstieg vs. } \\
\text { vor den Mahl- } \\
\text { zeiten }^{12}\end{array}$ & $\begin{array}{l}\text { keine Ände- } \\
\text { rung }^{13}\end{array}$ & $\mathrm{n}=0$ & $\begin{array}{l}\text { Anteil Pat. } \\
25 \%\end{array}$ \\
\hline \multirow{2}{*}{$\begin{array}{l}\text { Mathieu } \\
2014 \\
\text { 26-wöchige } \\
\text { offene RCT } \\
\text { (Treat-to- } \\
\text { Target) } \\
\mathrm{n}=177\end{array}$} & \multirow[t]{2}{*}{ IDeg + Met } & $\begin{array}{l}\text { IDeg + } \\
\text { Liraglutid } \\
+ \text { Met }\end{array}$ & $-0,74 \%^{14}$ & $58,0 \%$ & $-0,14$ & k. A. & $-2,8 \mathrm{~kg}^{15}$ & \multicolumn{2}{|c|}{$\begin{array}{l}\text { Ereignisse/Pat.-jahr } \\
\text { gesamt: } 1,00^{16} \\
\text { nächtliche: } 0,17^{17}\end{array}$} \\
\hline & & $\begin{array}{l}\text { IDeg + IAsp vor } \\
\text { größter Mahlzeit } \\
+ \text { Met }\end{array}$ & $-0,39 \%^{14}$ & $44,9 \%$ & $-0,04$ & k. A. & $+0,9 \mathrm{~kg}^{15}$ & $\begin{array}{l}\text { Ereignisse } \\
\text { gesamt: } 8 \\
\text { nächtlich: }\end{array}$ & $\begin{array}{l}\text { t.-jahr } \\
16 \\
11^{17}\end{array}$ \\
\hline
\end{tabular}

IGlar: Insulin glargin; IDeg: Insulin degludec; IDegLira: Fixkombination aus IDeg und Liraglutid; IAsp: Insulin aspart; RCT: randomisierte kontrollierte Studie; SH: Sulfonylharnstoff, Met: Metformin; OAD: orale Antidiabetika; k. A.: keine Angabe; Ereignisse/Pat.-jahr: Ereignisse/Patientenjahr; Vergl.gruppe: Vergleichsgruppe.

Zusätzlich: nicht-randomisierter Arm mit Ausgangs- $\mathrm{HbA}_{1 \mathrm{c}}<7,0 \%$ und Fortführung der vorherigen Therapie mit IDeg + Met* $(n=236)$.

${ }^{1}$ Mittlere Differenz: $-0,83 \mathrm{mmol} / \mathrm{l}(95 \% \mathrm{Cl}:-1,59$ bis $-0,07)$; $\mathrm{p}=0,021$

${ }^{2}$ Mittlere Differenz: $-7,6 \mathrm{~kg}(95 \% \mathrm{Cl}:-8,67$ bis $-6,57) ; \mathrm{p}<0,01$.

${ }^{3}$ Mittlere Differenz: $-0,74 \%$ ( $95 \% \mathrm{Cl}:-1,08$ bis $\left.-0,41\right)$; $p<0,001$.

${ }^{4}$ Mittlere Differenz: $-5,2 \mathrm{~kg}(95 \% \mathrm{Cl}:-6,7$ bis $-3,6)$; $\mathrm{p}<0,001$.

${ }^{5}$ Geschätzte Behandlungsdifferenz: $-1,19 \%(95 \% \mathrm{Cl}:-1,39$ bis $-0,99) ; \mathrm{p}<0,0001$.

${ }^{6}$ Geschätzte Odds ratio: 8,91 (95\% Cl: 5,45 bis 14,59); $<<0,0001$.

${ }^{7}$ Geschätzte Behandlungsdifferenz: $-1,28 \mathrm{mmol} / \mathrm{l}(95 \% \mathrm{Cl}:-1,70$ bis $-0,86)$; $\mathrm{p}<0,0001$.

${ }^{8}$ Ceschätzte Behandlungsdifferenz: $-0,57 \mathrm{mmol} / \mathrm{I}(95 \% \mathrm{Cl}:-0,94$ bis $-0,20) ; \mathrm{p}<0,0026$.

${ }^{9}$ Geschätzte Behandlungsdifferenz: $-3,11 \mathrm{~kg}(95 \% \mathrm{Cl}:-3,85$ bis $-2,37)$; $p<0,0001$.

${ }^{10}$ Geschätzte Behandlungsdifferenz: $-1,1 \%(95 \% \mathrm{Cl}:-1,3$ bis $-0,8) ; \mathrm{p}<0,0001$.

${ }^{11}$ Geschätzte Behandlungsdifferenz: $-0,73 \mathrm{mmol} / \mathrm{l}(95 \% \mathrm{Cl}:-1,19$ bis $-0,27)$; $\mathrm{p}=0,0019$.

${ }_{12}$ Geschätzte Behandlungsdifferenz: $-0,4 \mathrm{mmol} / \mathrm{l}(95 \% \mathrm{Cl}:-0,7$ bis $-0,0) ; \mathrm{p}=0,0260$.

${ }^{13}$ Geschätzte Behandlungsdifferenz: $-2,5 \mathrm{~kg}(95 \% \mathrm{Cl}:-3,2$ bis $-1,8) ; \mathrm{p}<0,0001$.

${ }^{14}$ Geschätzte mittlere Reduktion: $-0,32 \%$ ( $95 \% \mathrm{Cl}:-0,53$ bis $-0,12$ ); $\mathrm{p}=0,0024$.

${ }^{15}$ Geschätzte Behandlungsdifferenz: $-3,75 \mathrm{~kg}(95 \% \mathrm{Cl}:-4,70$ bis $-2,79)$; $\mathrm{p}<0,0001$.

${ }^{16}$ geschätzte Ratenrelation: 0,13 (95 Cl: 0,08 bis 0,21 ); $p<0,0001$.

${ }^{17}$ geschätzte Ratenrelation: 0,14 ( $95 \mathrm{Cl}: 0,05$ bis 0,40 ); $\mathrm{p}=0,0002$. 
tienten zu. In beiden Gruppen wurde im Rahmen des „Treat-toTarget"-Designs (Ziel: Nüchternblutzucker 4,0-5,0 mmol/l bzw. 72 - $90 \mathrm{mg} / \mathrm{dl}$ ) die IDeg-Dosis von $16 \mathrm{E} / \mathrm{Tag}$ auf im Mittel $45 \mathrm{E} / \mathrm{Tag}$ erhöht. Daher repräsentieren die Behandlungsdifferenzen den zusätzlichen Effekt von Liraglutid bei einer Zugabe zu Basalinsulin. Dabei entsprechen $45 \mathrm{E}$ IDeg in dem Fixkombinationspräparat 1,62 mg Liraglutid (1 Dosisschritt IDegLira: 1 E IDeg/0,036 mg Lira). Im Vergleich zu IDeg führte IDegLira zu signifikanten Reduktionen von $\mathrm{HbA}_{1 \mathrm{c}}$, Nüchternblutzucker, postprandialem Blutzucker und Körpergewicht, ohne das Hypoglykämierisiko zu erhöhen.

\section{Verträglichkeit der Intensivierung mit GLP-1-RA}

Eine Gesamtbetrachtung der Studien ergibt ein gehäuftes Auftreten von unerwünschten Ereignissen unter den drei GLP-1 RA im Vergleich zu den jeweiligen Kontrollgruppen. Dies ist vor allem auf gastrointestinale Ereignisse und hier insbesondere Übelkeit zurückzuführen. In der Studie von Mathieu et al. (2014) nahm der Anteil von Patienten mit Übelkeit im Verlauf deutlich ab und erreichte nach 26 Wochen 3\% ( $\bullet$ Abb.1a). Hinsichtlich der schwerwiegenden unerwünschten Ereignisse ergeben sich keine Hinweise auf eine schlechtere Verträglichkeit der GLP-1-RA.

Hervorzuheben ist das Ergebnis der doppelblinden Studie zu der Fixkombination IDegLira, in der eine ähnliche Rate bzgl. unerwünschter Ereignisse in der IDegLira-Gruppe versus IDeg gefunden werden konnte (Buse 2014). Zudem fällt auf, dass lediglich 6,5\% der mit IDegLira behandelten Patienten von Übelkeit betroffen waren $(\bullet$ Abb. 1b). Ein wesentlicher Unterschied zu den anderen Studien bestand in der 2-mal wöchentlichen Dosistitration in kleinen Schritten (jeweils \pm 2 Dosisschritte, entsprechend 0,072 mg) ausgehend von 0,6 mg Liraglutid (Mathieu et al. 2014). Eine Lösung des Problems der gastrointestinalen Nebenwirkungen könnte daher in kleineren Titrationsschritten im Vergleich zum bisher üblichen Vorgehen liegen.

\section{Blutdruckreduktion im Rahmen der Intensivierung mit GLP-1-RA}

In den Publikationen zu vier randomisierten, kontrollierten Studien werden Ergebnisse zum Blutdruck berichtet. Es zeigten sich jeweils Reduktionen des systolischen Blutdrucks mit Differenzen zu den Vergleichsgruppen von 4-5 mm Hg. Eine signifikante Reduktion des diastolischen Blutdrucks konnte bisher nur in der placebokontrollierten Studie von Buse et al. (2011) gezeigt werden, die zugleich einen signifikanten Anstieg des Pulses um 3,0 Schläge/ Minute (95\% CI: 0,8 bis 5,2; p < 0,01) dokumentierte. Dies steht im Einklang mit früheren Untersuchungen (Fonseca 2014).

\section{Reduktion der Insulindosis}

In Erwartung des zusätzlichen blutzuckersenkenden Effekts und eines geringeren Insulinbedarfs wurden in den meisten Studien die Insulindosierungen bereits zum Zeitpunkt der Einleitung der Therapie mit GLP-1-RA reduziert. Das Vorgehen war jedoch unterschiedlich. So differenzierte nicht jede Studie bei der Dosisreduktion zwischen den Ausgangs-HbA 1 -Werten. Zudem lag die Spanne der Reduktion der Basalinsulindosis zwischen $10 \%$ und $30 \%$.

\section{Zeitpunkt des Therapiebeginns mit GLP-1-RA \\ $\nabla$}

Da ein Großteil der Wirkung der GLP-1-RA von der Fähigkeit der $ß$-Zellen abhängt, Insulin freizusetzen, stellt sich die Frage nach dem optimalen Zeitpunkt für die erstmalige Gabe von GLP-1-RA bei Typ-2-Diabetes. Der Vergleich von explorativen Daten aus zwei Studien ergab gegenläufige Hinweise zur Wirksamkeit von GLP-1-RA in Abhängigkeit von der Diabetesdauer (Penfornis 2013, Rosenstock 2012).

Gemäß Subgruppenanalyse zur placebokontrollierten Studie zu Exenatid $(n=259)$ (Buse 2011) wurde bei einer Diabetesdauer von < 9 Jahren keine signifikante Reduktion des $\mathrm{HbA}_{1 \mathrm{c}}$ gegenüber einer optimierten Insulin-glargin-Therapie erreicht, während es bei längerer Diabetesdauer zu signifikanten Vorteilen kam (Rosenstock 2012). Zudem war die $\mathrm{HbA}_{1 \mathrm{c}}$-Reduktion unabhängig vom Ausgangs- $\mathrm{HbA}_{1 \mathrm{c}}$ und geringer bei Patienten mit hohem BMI $>36 \mathrm{~kg} / \mathrm{m}^{2}$. Eine signifikante Gewichtsreduktion im Vergleich zu Placebo wurde in allen untersuchten Subgruppen erreicht.

In der französischen Beobachtungsstudie EVIDENCE erhielten 2433 Patienten für mehr als ein Jahr Liraglutid zusätzlich zu oralen Antidiabetika und/oder Insulin. Hier war die $\mathrm{HbA}_{1 \mathrm{c}}$-Reduktion am stärksten bei kurzer Diabetesdauer und bei wenigen Antidiabetika in der Vortherapie (Penfornis 2013). In einer Posthoc-Analyse von Daten aus dem Phase-3-Studienprogramm zu Liraglutid war die $\mathrm{HbA}_{1 \mathrm{c}}$-Reduktion etwas stärker bei kürzerer Diabetesdauer, wenn 1,2 mg Liraglutid appliziert wurden (Seufert 2013). Unter $1,8 \mathrm{mg}$ Liraglutid war kein signifikanter Einfluss der Diabetesdauer auf die $\mathrm{HbA}_{1 \mathrm{c}}$-Reduktion festzustellen.

\section{Patienten mit Adipositas und schwerer Insulinresistenz $\nabla$}

Zwei Studien untersuchten Exenatid (Nayak 2010) oder Liraglutid (Lane 2014) bei adipösen Patienten mit Typ-2-Diabetes und hoher Insulindosis.

In der prospektiven Beobachtungsstudie zu Exenatid zeigten sich im Verlauf von 6 Monaten bei den 160 Patienten eine durchschnittliche Gewichtsreduktion um - 10,7 kg und ein nahezu unveränderter $\mathrm{HbA}_{1 \mathrm{c}}$ von 8,6\% gegenüber 8,8\% als Ausgangsbefund (Nayak 2010). Zugleich konnte jedoch die Insulindosis bereits in den ersten 3 Monaten im Mittel von 144 auf 51 E/Tag reduziert werden einschließlich 39 Patienten (24\%), bei denen Insulin abgesetzt worden war.

Die Ergebnisse der randomisierten kontrollierten Studie zu Liraglutid sind in der $\odot$ Tab. 3 dargestellt (Lane 2014). Bei einer Reduktion der Insulindosis in der Liraglutid-Gruppe um durchschnittlich $34 \%$ versus nicht signifikantem Anstieg von $171 \mathrm{E}$ auf $178 \mathrm{E}$ bei Intensivierung der Insulintherapie war die Gewichtsreduktion nach 6 Monaten mit $-5,3 \mathrm{~kg}$ versus Baseline $(\mathrm{p}<0,0001)$ weniger stark ausgeprägt als in der Studie von Nayak et al. (2010); jedoch wurde eine signifikante Reduktion des $\mathrm{HbA}_{1 \mathrm{c}}$ gegenüber dem Ausgangswert von 7,80\% auf 7,15\% erreicht $(\mathrm{p}<0,0001)$ (Lane 2014).

\section{Persistenz \\ $\nabla$}

In einer US-amerikanischen Untersuchung zu Patienten mit Typ2-Diabetes, die erstmals Basalinsulin oder Exenatid erhielten, war die Persistenz nach dem ersten Behandlungsjahr mit 28,7\% gering (Cooke 2010). Exenatid wurde im Mittel nach 7,6 Monaten beendet im Vergleich zu jeweils 7,8 Monaten bei Insulin glargin und Insulin detemir $(\mathrm{p}=\mathrm{n}$. s.) sowie durchschnittlich 5,6 Monaten bei NPH-Insulin $(p<0,001)$. In einer anderen Studie war die Persistenz deutlich besser, mit einem Vorteil für Exenatid im Vergleich zu Insulin glargin (68 $\pm 29 \%$ vs. $58 \pm 28 \%$; p < 0,001) (Fabunmi 2009). Auch die französische prospektive Beobachtungs- 
studie zu Liraglutid ergibt Hinweise, dass eine deutlich höhere Persistenz als in der US-amerikanischen Untersuchung erreicht werden kann, da nach einem Jahr noch 77,6\% der Patienten an der Studie teilnahmen (Penfornis 2013).

Die drei randomisierten kontrollierten Studien, die Exenatid, Liraglutid oder Albiglutid mit einem kurz wirksamen Insulinanalogon zur Intensivierung einer Basalinsulintherapie verglichen, wiesen relativ hohe Raten an Patienten auf, die nach 30 bzw. 26 Wochen noch in der Studie waren. Mit $84 \%$ (Exenatid) versus $86 \%$ (Insulin lispro), 86\% (Liraglutid) versus 84\% (Insulin aspart) bzw. $91 \%$ (Albiglutid) versus $94 \%$ (Insulin lispro) ergibt sich kein Hinweis auf einen Unterschied bei der Persistenz zwischen GLP-1-RA und kurz wirksamem Insulinanalogon (Diamant 2014, Mathieu 2014, Rosenstock 2014). Auffallend war jedoch, dass sich in der Studie von Diamant et al. (2014) 29 Patienten (9,3\%) in der Insulin-lisproGruppe für ein vorzeitiges Studienende entschieden im Vergleich zu 18 Patienten (5,7\%) in der Exenatid-Gruppe (Diamant 2014). Unerwünschte Ereignisse waren dagegen in den GLP-1-RA-Gruppen häufiger für Abbrüche verantwortlich als in den Gruppen der kurz wirksamen Insulinanaloga (5,4\% Exenatid, 5,7\% Liraglutid und 5,3\% Albiglutid versus 2,6\%, 1,1\% und 0,4\% Insulinanaloga). Es dürfte daher von entscheidender Relevanz für eine gute Persistenz im Behandlungsalltag sein, dass die Betreuung der Patienten in Bezug auf gastrointestinale Ereignisse optimiert ist.

\section{Therapietreue}

$\nabla$

Bei einer unzureichenden Therapietreue von Patienten mit Typ2-Diabetes steigt der $\mathrm{HbA}_{1 \mathrm{c}}$ und es kommt zu gehäuften Arztbesuchen, Behandlungen in der Notaufnahme sowie Hospitalisierungen (Di Bonaventura 2014). Die Gründe einer reduzierten Therapieadhärenz sind vielschichtig und umfassen u. a. die Komplexität der Behandlung, Nebenwirkungen wie Gewichtszunahme und Hypoglykämie und die wahrgenommene Wirksamkeit (García-Pérez 2013). Bei den GLP-1-RA stehen den gastrointestinalen Nebenwirkungen zahlreiche Faktoren gegenüber, die von Vorteil für die Therapieadhärenz sein können. Dazu gehören insbesondere das im Vergleich zu kurz wirksamen Insulinen geringere Hypoglykämierisiko, die deutliche Gewichtsreduktion und eine relativ geringe Komplexität der Behandlung (weniger Blutzuckerkontrollen, ggf. geringere Applikationshäufigkeit).

Bei Liraglutid könnte sich die nur 1-mal tägliche Gabe, die zudem von Mahlzeiten unabhängig ist (beliebiger Zeitpunkt, jedoch vorzugsweise immer zur gleichen Tageszeit) positiv im Vergleich zu Exenatid auswirken, das 2-mal täglich jeweils innerhalb von 60 Minuten vor Mahlzeiten angewendet werden muss (Santoleri 2014). Außerdem ergeben sich für die im September 2014 neu zugelassene Fixkombination von Liraglutid und IDeg (Xultophy ${ }^{\circledR}$ ) positive Aspekte für die Therapietreue der Patienten. So wird für eine Intensivierung der Basalinsulintherapie keine zusätzliche Injektion mehr benötigt, und es waren nur 6,5\% der Patienten von Übelkeit betroffen (Buse 2014).

\section{Abwägung Exenatid versus Liraglutid versus Albiglutid $\nabla$}

Bei den Abwägungen zur Intensivierung einer Therapie mit Basalinsulinen sollten Unterschiede zwischen den GLP-1-RA berücksichtigt werden, um die Therapie besser auf den jeweiligen Patienten auszurichten (Lund 2014). Grundsätzlich ermöglicht die 2-mal tägliche Gabe von Exenatid im Vergleich zu 1-mal täg- lich Liraglutid eine stärkere Reduktion der postprandialen Blutzuckerwerte bei zwei von drei Hauptmahlzeiten am Tag, die postprandialen Blutzuckerwerte nach dem Mittagessen unterschieden sich nicht statistisch signifikant (Buse 2009). Dies gelingt durch eine stärkere Hemmung der Magenentleerung nach der Injektion (Meier 2012, Montanya 2012). Dagegen wurde mit Liraglutid eine stärkere Reduktion des Nüchternblutzuckers erreicht (Buse 2009). Der Effekt auf den Nüchternblutzucker beruht primär auf insulinotropen Wirkungen und der Reduktion der Glucagonsekretion (Meier 2012).

In der LEAD-6-Studie waren in der Behandlungswoche 26 noch 2,5\% der mit Liraglutid behandelten Patienten versus 8,6\% der mit Exenatid behandelten Patienten von Übelkeit betroffen (Buse 2009). Bis zum Abfall der Übelkeitsrate unter $10 \%$ vergingen in der Liraglutid-Gruppe 6 Wochen im Vergleich zu 22 Wochen in der Exenatid-Gruppe (Buse 2009). Die Liraglutid-Gruppe wies zudem insgesamt eine höhere Therapiezufriedenheit auf als die Exenatid-Gruppe (Buse 2009).

In der 32-wöchigen HARMONY-7-Studie wurden mit Liraglutid (Startdosis 0,6 mg, Titration bis auf $1,8 \mathrm{mg}$ ab Woche 3 ) häufiger das $\mathrm{HbA}_{1 \mathrm{c}}$-Ziel und eine im Mittel um 1,55 kg stärkere Gewichtsreduktion erreicht als mit Albiglutid (Startdosis $30 \mathrm{mg}$, Steigerung auf $50 \mathrm{mg}$ in Woche 6) (Pratley 2014). Die erhöhte Übelkeitsrate in der Liraglutid-Gruppe ist möglicherweise auf die schnellere Dosistitration zurückzuführen.

\section{Abwägung GLP-1-RA versus kurz wirksame Insulinanaloga}

Anhand der präsentierten Studienergebnisse aus drei direkten Vergleichsstudien und drei weiteren randomisierten kontrollierten Studien (Buse 2011, Diamant 2014, Lahtela 2014, Mathieu 2014, Buse 2014, Rosenstock 2014) sowie der darüber hinausgehenden Eigenschaften lassen sich Vorteile für die jeweilige Option zur Intensivierung einer Therapie mit Basalinsulinen darstellen ( $\bullet$ Tab.4).

Kurz wirksame Insulinanaloga können von Vorteil sein, wenn ein Patient besonders gut für eine intensivierte konventionelle Insulintherapie (ICT) geeignet ist und/oder ein stark variierender Lebensstil inklusive Essgewohnheiten vorliegt. Durch eine situationsbedingte Anpassung der Insulindosis können bei derartigen Patienten stärkere Blutzuckerspitzen vermieden werden. Für die Reduktion von postprandialen Blutzuckerwerten durch kurzwirksame Insulinanaloga liegen Hinweise vor, dass im Vergleich zu Humaninsulin kardiovaskuläre Parameter verbessert und Folgekomplikationen vermieden werden können (Kress 2012, Rathmann 2013).

Weitere Aspekte, die für eine Intensivierung mit kurz wirksamen Insulinanaloga sprechen, sind die geringeren Raten gastrointestinaler Nebenwirkungen und hierdurch bedingter Therapieabbrüche zu Behandlungsbeginn, weniger Einschränkungen bzgl. besonderer Patientengruppen und die Möglichkeit zur genaueren Dosistitration ( $\bullet$ Tab.4).

Für eine Intensivierung mit GLP-1-RA anstelle von kurz wirksamen Insulinanaloga sprechen die deutlich besseren Gewichtsverläufe, weniger Hypoglykämien, das Vorliegen von deutlich mehr Faktoren, die sich positiv auf die Therapietreue des Patienten auswirken können, weniger Blutzuckermessungen, die Dosisreduktion von Insulin mit eventueller Möglichkeit zum Absetzen von Insulin und eine höhere Therapiezufriedenheit. Letztere wurde in der Studie von Diamant et al. (2014) unter Exenatid im Vergleich zu Insulin li- 
GLP-1-RA vs. kurz wirksame Insulinanaloga

\begin{tabular}{|c|c|}
\hline $\begin{array}{l}\mathrm{HbA}_{1 \mathrm{c}}-\text { Reduk- } \\
\text { tion/ } \mathrm{HbA}_{1 \mathrm{c}} \\
\leq 7,0 \%\end{array}$ & $\begin{array}{l}\left.\text { - Vergleichbare } \mathrm{HbA}_{1 \mathrm{c}} \text {-Reduktion (ca. } 1 \%\right)^{1-6} \\
\text { - Vergleichbarer prozentualer Anteil an Patienten mit } \mathrm{HbA}_{1 \mathrm{c}} \leq 7 \%{ }^{1-6}\end{array}$ \\
\hline $\begin{array}{l}\text { Blutzucker } \\
\text { nüchtern (nüBZ) }\end{array}$ & $\begin{array}{l}\text { - GLP-1-RA mit Effekt auf nüBZ (Liraglutid > Exenatid }{ }^{7} \text { ) } \\
\text { - Exenatid vs. Insulin lispro im Vorteil (Behandlungsdifferenz-0,64 mmol//2) }\end{array}$ \\
\hline $\begin{array}{l}\text { Blutzucker } \\
\text { postprandial } \\
\text { (ppBZ) }\end{array}$ & $\begin{array}{l}\text { - Kurz wirksame Insulinanaloga vs. Exenatid oder Liraglutid mit Vorteil bei einer von drei } \\
\text { Hauptmahlzeiten }{ }^{1,2} \\
\text { - Kurz wirksames Insulin ermöglicht gezielte Beeinflussung des Blutzuckers bei jeder Mahlzeit }\end{array}$ \\
\hline Hypoglykämien & $\begin{array}{l}\text { - GLP-1-RA mit geringerem Hypoglykämierisiko (z. B. } 1 \text { vs. } 8 \text { Hypoglykämien pro Pat.-jahr) 1,2,6 } \\
\text { - Zugabe von GLP-1-RA oder kurz wirksamem Insulin erfordert ggf. Dosisanpassung des Basal- } \\
\text { insulins. }{ }^{1,2}\end{array}$ \\
\hline Körpergewicht & $\begin{array}{l}\text { - Effekt von Exenatid und Liraglutid ca. } 3 \mathrm{~kg}^{1-5} \\
\text { - Ca. } 4 \text { kg Differenz vs. kurz wirksames Insulin }{ }^{1-2}\end{array}$ \\
\hline $\begin{array}{l}\text { Systolischer Blut- } \\
\text { druck }\end{array}$ & - Exenatid und Liraglutid: Reduktion um 4-5 mmHg $\mathrm{mb}^{2-4}$ \\
\hline Nebenwirkungen & $\begin{array}{l}\text { - GLP-1-RA: GI-UE, aber Hinweise auf relativ wenig SUE1 -3,5,6 } \\
\text { - Kurz wirksames Insulin: zu Beginn weniger GI-UE (um ca. } 3 \text { von } 10 \text { behandelten Pat.)2 }\end{array}$ \\
\hline $\begin{array}{l}\text { Therapie- } \\
\text { persistenz }\end{array}$ & $\begin{array}{l}\text { - Unter kurz wirksamem Insulin zu Beginn der Therapie weniger Abbrüche wegen UE (um ca. } \\
3 \text { von } 100 \text { Pat.) })^{1,2,6}\end{array}$ \\
\hline $\begin{array}{l}\text { Therapie- } \\
\text { adhärenz }\end{array}$ & $\begin{array}{l}\text { - Unter GLP-1-RA mehr fördernde Faktoren: Körpergewicht, Hypoglykämien, Komplexität, } \\
\text { weniger Blutzuckermessungen, höhere Therapiezufriedenheit }{ }^{2}\end{array}$ \\
\hline Sonstiges & $\begin{array}{l}\text { Kurz wirksame Insulinanaloga: } \\
\text { - Weniger Einschränkungen bzgl. besonderer } \\
\text { Patientengruppen (z. B. Kinder, Pat. mit } \\
\text { Niereninsuffizienz) } \\
\text { - Dosistitration ohne Maximaldosis möglich }{ }^{10} \\
\text { - Mischinsuline verfügbar }\end{array}$ \\
\hline
\end{tabular}

UE: unerwünschtes Ereignis; SUE: schwerwiegende UE; Gl: gastrointestinal; Pat.: Patient;

${ }^{1}$ Mathieu et al. 2014; ${ }^{2}$ Diamant et al. 2014; ${ }^{3}$ Buse 2011; ${ }^{4}$ Lahtela 2014; ${ }^{5}$ Buse $2014 ;{ }^{6}$ Rosenstock 2014; $;$ Buse 2009; ${ }^{8}$ de Wit 2014; ${ }^{9}$ Li 2012; ${ }^{10}$ Owens 2013; ${ }^{11}$ Vardar 2007

spro und in einer schwedischen Beobachtungsstudie zur Intensivierung einer Insulintherapie mit Liraglutid $(n=40)$ oder Exenatid $(\mathrm{n}=21$ ) gezeigt (Lind 2012). Bei bestimmten Patienten kann zudem die insbesondere unter Liraglutid zu erwartende stärkere Reduktion des Nüchternblutzuckers relevant sein. Für IDegLira können Vorteile der Fixkombination (nur eine Injektion pro Tag) und die möglicherweise geringere Übelkeitsrate sprechen.

\section{Fazit}

In jüngster Zeit wurden mehrere randomisierte, kontrollierte Studien zur Intensivierung einer Basalinsulintherapie mit drei GLP-1RA publiziert. Demnach ermöglichen GLP-1-RA eine kombinierte Blutzuckerreduktion (postprandial und nüchtern) mit positiven Effekten auf den $\mathrm{HbA}_{1 \mathrm{c}}$-Wert, das Hypoglykämierisiko, das Körpergewicht, die Insulindosis, den systolischen Blutdruck und die Therapiezufriedenheit der Patienten. Außerdem sind weniger Blutzuckermessungen als bei einer Therapie mit kurz wirksamen Insulinanaloga notwendig. Inwieweit auch die Therapietreue der Patienten verbessert werden kann, ist zum jetzigen Zeitpunkt lediglich auf der Basis theoretischer Erwägungen zu beantworten. Zudem konnten die in früheren Studien gezeigten Einflüsse auf den Lipidstoffwechsel bisher nicht anhand der hier präsentierten Studien zur Kombination mit Basalinsulin bestätigt werden.

Trotz der vielschichtigen positiven Eigenschaften von GLP-1-RA gibt es Gründe, die für eine Intensivierung mit kurz wirksamen Insulinanaloga sprechen. Dazu gehören die Möglichkeit, Blutzuckerspitzen zu allen drei Hauptmahlzeiten jeweils gezielt zu behandeln, die deutlich geringere Rate an gastrointestinalen unerwünschten Ereignissen zu Therapiebeginn und weniger Ein-
Tab. 4 Aspekte für die Abwägung zwischen GLP-1-RA und kurz wirksamen Insulinanaloga bei der Intensivierung einer Therapie mit Basalinsulin.

Interessenkonflikte: Prof. Dr. B. Gallwitz führt Vortragstätigkeiten für AstraZeneca, Berlin-Chemie, Boehringer-Ingelheim, Bristol Myers Squibb, Novo Nordisk, Lilly und Sanofi durch, ist Mitglied in Advisory Boards bei AstraZeneca, Boehringer-Ingelheim, Lilly MSD, Novo Nordisk und erhält Forschungsunterstützung von Novo Nordisk.

Prof. Dr. med. Stephan Matthaei führt Vortragstätigkeiten für Abbott, AstraZeneca, Boehringer, Lilly, MSD, Novo Nordisk und Sanofi durch, ist Mitglied in Advisory Boards bei Abbott, AstraZeneca, Boehringer, MSD, Novo Nordisk und Roche und erhält Forschungsunterstützung von Roche. 


\section{Literatur}

1 Agersø H, Jensen LB, Elbrond B et al. The pharmacokinetics pharmacodynamics, safety and tolerability of NN2211, a new long-acting GLP-1 derivative, in healthy men. Diabetologia 2002; 45: 195-202

2 Ahrén B. Insulin plus incretin: A glucose-lowering strategy for type 2diabetes. World J Diabetes 2014; 5: 40-51

3 Alves C, Batel-Marques F, Macedo AF. A meta-analysis of serious adverse events reported with exenatide and liraglutide: acute pancreatitis and cancer. Diabetes Res ClinPract 2012; 98: 271-84

4 Arnolds S, Dellweg S, Clair J et al. Further improvement in postprandial glucose control with addition of exenatide or sitagliptin to combination therapy with insulin glargine and metformin: a proof-of-concept study. Diabetes Care 2010; 33: 1509-1515

5 Astrup A, Carraro R, Finer $N$ et al. NN8022-1807 Investigators. Safety, tolerability and sustained weight loss over 2 years with the once-daily human GLP-1 analog, liraglutide. Int J Obes (Lond) 2012; 36: 843 - 854

6 Balena R, Hensley IE, Miller S et al. Combination therapy with GLP-1 receptor agonists and basal insulin: a systematic review of literature. Diabetes Obes Metab 2013; 15: 485-502

7 Bawa T, Dhingra V, Malhotra $N$ et al. Clinical experience with exenatide in obese North Indian patients with type 2 diabetes mellitus. Indian J EndocrinolMetab 2013; 17: 91 -94

8 Bergenstal RM, Wysham C, Macconell L et al. Efficacy and safety of exenatide once weekly versus sitagliptin or pioglitazone as an adjunct to metformin for treatment of type 2 diabetes (DURATION-2): a randomised trial. Lancet 2010; 376 (9739): $431-439$

9 Brown DX, Evans M. Choosing between GLP-1 Receptor Agonists and DPP-4 Inhibitors: A Pharmacological Perspective. J NutrMetab 2012; 2012: 381713 Epub 2012 Oct 18 DOI: 10.1155/2012/381713

10 Buse J, RosenstockJ, Sesti G et al. Liraglutide once a day versus exenatide twice a day for type 2 diabetes: a 26-week randomised, parallel-group, multinational, open-label trial (LEAD 6). Lancet 2009; 374: 39-47

11 Buse JB, Bergenstal RM, Glass LC et al. Use of twice-daily exenatide in Basal insulin-treated patients with type 2 diabetes: a randomized, controlled trial. Ann Intern Med 2011; 154: 103-112

12 Buse JB, Vilsbøll T, Thurman J et al. NN9068-3912 (DUAL-II) Trial Investigators. Contribution of liraglutide in the fixed-ratio combination of insulin degludec and liraglutide (IDegLira). Diabetes Care 2014; 37: $2926-2933$

13 Cooke CE, Lee HY, Tong YP et al. Persistence with injectable antidiabetic agents in members with type 2 diabetes in a commercial managed care organization. Curr Med Res Opin 2010; 26: 231 - 238

14 De Wit HM, Vervoort GM, Jansen HJ et al. Liraglutide reverses pronounced insulin-associated weight gain, improves glycaemic control and decreases insulin dose in patients with type 2 diabetes: a 26 week, randomised clinical trial (ELEGANT). Diabetologia 2014; 57: 1812-1819

15 Diamant M, Nauck MA, Shaginian R et al. 4B Study Group. Glucagonlike peptide-1 receptor agonist or bolus insulin with optimized basal insulin in type 2 diabetes. Diabetes Care 2014; 37: 2763-2773

16 DiBonaventura $M$, Wintfeld $N$, Huang J et al. The association between nonadherence and glycated hemoglobin among type 2 diabetes patients using basal insulin analogs. Patient Prefer Adherence 2014; 8 : $873-882$

17 Drucker DJ, Nauck MA. The incretin system: glucagon-like peptide-1 receptor agonists and dipeptidyl peptidase- 4 inhibitors in type 2 diabetes. Lancet 2006; 368: 1696-1705

18 Elbrønd B, Jakobsen G, Larsen $S$ et al. Pharmacokinetics, pharmacodynamics, safety, and tolerability of a single-dose of NN2211, a longacting glucagon-like peptide 1 derivative, in healthy male subjects. Diabetes Care 2002; 25: 1398 - 1404

19 Fabunmi R, Nielsen LL, Quimbo R et al. Patient characteristics, drug adherence patterns, and hypoglycemia costs for patients with type $2 \mathrm{di}-$ abetes mellitus newly initiated on exenatide or insulin glargine. Curr Med Res Opin 2009; 25: 777-786

20 Faillie JL, Azoulay L, Patenaude $V$ et al. Incretin based drugs and risk of acute pancreatitis in patients with type 2 diabetes: cohort study. BM] 2014: 348:g2780. DOI: 10.1136/bmj.g2780

21 Flint A, Kapitza C, Hindsberger C et al. The once-daily human glucagonlike peptide-1 (GLP-1) analog liraglutide improves postprandial glucose levels in type 2 diabetes patients. AdvTher 2011;28: 213-226

22 Fonseca VA, Devries JH, Henry RR et al. Reductions in systolic blood pressure with liraglutide in patients with type 2 diabetes: insights from a patient-level pooled analysis of six randomized clinical trials. J Diabetes Complications 2014; 28: 399-405
23 García-Pérez LE, Álvarez M, Dilla T et al. Adherence to Therapies in Patients with Type 2 Diabetes. Diabetes Ther 2013; 4: 175-194

24 Gough SC, Bode B, Woo V et al. NN9068-3697 (DUAL-I) trial investigators. Efficacy and safety of a fixed-ratio combination of insulin degludec and liraglutide (IDegLira) compared with its components given alone: results of a phase 3, open-label, randomized, 26-week, treat-to-target trial in insulin naïve patients with type 2 diabetes. Lancet Diabetes Endocrinol 2014; 2: 885-893

25 Gutzwiller JP, Drewe J, Göke B et al. Glucagon-like peptide-1 promotes satiety and reduces food intake in patients with diabetes mellitus type 2. Am J Physiol 1999; 276: R1541 - R1544

26 Helleberg $\mathrm{H}$. Metabolism and Excretion of [Pal-3H]-Liraglutide in Human Healthy Subjects. Diabetes 2008; 57 (Suppl 1): 2107-PO

27 Holst JJ, Vilsbøll T. Combining GLP-1 receptor agonists with insulin: therapeutic rationales and clinical findings. Diabetes Obes Metab 2013; 15: 3-14

28 Inzucchi SE, Bergenstal RM, Buse JB et al. Management of hyperglycemia in type 2 diabetes 2015: a patient-centered approach. Update to a position statement of the American Diabetes Association and the European Association for the Study of Diabetes. Diabetes Care 2015; 38: 140 149

29 Jendle J, Martin SA, Milicevic Z. Insulin and GLP-1 analog combinations in type 2 diabetes mellitus: a critical review. Expert Opin Investig Drugs 2012; 21: $1463-1474$

30 Kress S, Kostev K, Dippel FW et al. Micro- and macrovascular outcomes in Type 2 diabetic patients treated with insulin glulisine or human regular insulin: a retrospective database analysis. Int J Clin Pharmacol Ther 2012; 50: $821-829$

31 Kumar A. Insulin degludec/liraglutide: innovation-driven combination for advancement in diabetes therapy. Expert Opin Biol Ther 2014; 14 $869-878$

32 Lahtela J, Ahmann A, Rodbard $\mathrm{H}$ et al. Efficacy and safety of liraglutide vs placebo when added to basal insulin analogues in subjects with type 2 diabetes (LIRA-ADD2BASAL): a randomized, placebo-controlled trial. Diabetologia 2014; 57 (Suppl 1): S21 (Abstr. 37)

33 Landgraf $R$, Kellerer $M$, Fach $E$ et al. Praxisempfehlungen DDG/DGIM. Therapie des Typ-2-Diabetes. Diabetologie 2013; 8: S146-S158

34 Lane W, Weinrib S, Rappaport J et al. The effect of addition of liraglutide to high-dose intensive insulin therapy: a randomized prospective trial. Diabetes Obes Metab 2014; 16: 827-832

35 Li CJ, Li J, Zhang QM et al. Efficacy and safety comparison between liraglutide as add-on therapy to insulin and insulin dose-increase in Chinese subjects with poorly controlled type 2 diabetes and abdominal obesity. Cardiovasc Diabetol 2012; 11: 142

$36 \mathrm{Li}$ L, Shen J, Bala MM et al. Incretin treatment and risk of pancreatitis in patients with type 2 diabetes mellitus: systematic review and meta-analysis of randomised and non-randomised studies. BMJ 2014; 348: g2366. DOI: 10.1136/bmj.g2366

37 Lind $M$, Jendle J, Torffvit $O$ et al. Glycagon-like peptide 1 (GLP-1) analogue combined with insulin reduces HbA1c and weight with low risk of hypoglycemia and high treatment satisfaction. Prim Care Diabetes 2012: 6: $41-46$

38 Linnebjerg H, Park S, Kothare PA et al. Effect of exenatide on gastric emptying and relationship to postprandial glycemia in type 2 diabetes. Regul Pept 2008; 151: 123-129

39 Liu R, Li L, Chen Y et al. Effects of glucagon-like peptide-1 agents on left ventricular function: systematic review and meta-analysis. Ann Med 2014; 46: 664-671

40 Lund A, Knop FK, Vilsbøll T. Glucagon-like peptide-1 receptor agonists for the treatment of type 2 diabetes: differences and similarities. Eur J Intern Med 2014; 25: 407-414

41 Mathieu C, Rodbard HW, Cariou B et al. A comparison of adding liraglutide versus a single daily dose of insulin aspart to insulin degludec in subjects with type 2 diabetes (BEGIN: VICTOZA ADD-ON). Diabetes Obes Metab 2014; 16: 636-644

42 Meier IJ. GLP-1 receptor agonists for individualized treatment of type 2 diabetes mellitus. Nat Rev Endocrinol 2012; 8: 728 - 742

43 Merker L, Wilhelm B, Kaiser M et al. Insulin degludec bei insulinnaiven Typ-2-Diabetikern: weniger nächtliche Hypoglykämien. Diabetologie, Stoffwechsel und Herz 2013; 22: 377-384

44 Montanya E. A comparison of currently available GLP-1 receptor agonists for the treatment of type 2 diabetes. Expert Opin Pharmacother 2012; $13: 1451-1467$ 
45 Nauck MA, Niedereichholz U, Ettler $R$ et al. Glucagon-like peptide 1 inhibition of gastric emptying outweighs its insulinotropic effects in healthy humans. Am J Physiol 1997; 273: E981 -E988

46 Nayak UA, Govindan J, Baskar $V$ et al. Exenatide therapy in insulintreated type 2 diabetes and obesity. OJM 2010; 103: 687-694

47 Nikfar S, Abdollahi M, Salari P. The efficacy and tolerability of exenatide in comparison to placebo; a systematic review and meta-analysis of randomized clinical trials. J Pharm Pharm Sci 2012; 15: 1 - 30

48 Okerson T, Chilton RJ. The cardiovascular effects of GLP-1 receptor agonists. Cardiovasc Ther 2012; 30: e146-e155

49 Owens $D R$. Stepwise intensification of insulin therapy in Type 2 diabetes management-exploring the concept of the basal-plus approach in clinical practice. Diabet Med 2013; 30: 276-288

50 Penfornis A, Gourdy P, Martinez L et al. Diabetes duration and background diabetes therapies in preceding liraglutide treatment response: data from the post-marketing EVIDENCE study. Diabetologia 2013; 56 (Suppl 1): S356 (Abstr. 894)

51 Pottegård A, Bjerregaard BK, Larsen MD et al. Use of exenatide and liraglutide in Denmark: a drug utilization study. Eur J Clin Pharmacol 2014; 70: 205-214

52 Pratley RE, Nauck M, Bailey $T$ et al. Liraglutide versus sitagliptin for patients with type 2 diabetes who did not have adequate glycaemic control with metformin: a 26-week, randomised, parallel-group, open-label trial. Lancet 2010; 375: 1447 -1456

53 Pratley RE, Nauck MA, Barnett AH et al. for the HARMONY 7 study group. Once-weekly albiglutide versus once-daily liraglutide in patients with type 2 diabetes inadequately controlled on oral drugs (HARMONY 7): a randomized, open-label, multicentre, non-inferiority phase 3 study. Lancet Diabetes Endocrinol 2014; 2: 289-297

54 Rathmann W, Kostev $K$. Lower incidence of recorded cardiovascular outcomes in patients with type 2 diabetes using insulin aspart vs. those on human regular insulin: observational evidence from general practices. Diabetes Obes Metab 2013; 15: 358-363

55 Rodbard HW, Cariou B, Zinman B et al. Comparison of insulin degludec with insulin glargine in insulin-naive subjects with Type 2 diabetes: a 2-year randomized, treat-to-target trial. Diabet Med 2013b; 30: $1298-1304$

56 Rodbard HW, Visco VE, Andersen $H$ et al. Treatment intensification with stepwise addition of prandial insulin aspart boluses compared with full basal-bolus therapy (FullSTEP Study): a randomised, treat-to-target clinical trial. Lancet 2013a; DOI: 10.1016/S2213-8587(13)70090-1
57 Rosenstock J, Fonseca VA, Gross JL et al. Advancing basal insulin replacement in type 2 diabetes inadequately controlled with insulin glargine plus oral agents: a comparison of adding albiglutide, a weekly GLP-1 receptor agonist, versus thrice-daily prandial insulin lispro. Diabetes Care 2014; 37: 2317-2325

58 Rosenstock J, Shenouda SK, Bergenstal RM et al. Baseline factors associated with glycemic control and weight loss when exenatide twice daily is added to optimized insulin glargine in patients with type 2 diabetes. Diabetes Care 2012; 35: 955 - 958

59 Santoleri F, Sorice P, Lasala R et al. Liraglutide vs exenatide: patient adherence, medication persistence and economic evaluation in the treatment of type 2 diabetes mellitus. Pharmacol Pharmacy 2014; 5: 332 339

60 Seufert J, Gallwitz B. The extra-pancreatic effects of GLP-1 receptor agonists: a focus on the cardiovascular, gastrointestinal and central nervous systems. Diabetes Obes Metab 2014; 16: 673-688

61 Seufert JR, Bailey T, Svendsen C et al. The effect of liraglutide on HbA1C and body weight is largely independent of baseline diabetes duration. Diabetologia 2013; 56 (Suppl 1): S356 (Abstr. 895)

62 Sun F, Yu K, Yang Z et al. Impact of GLP-1 receptor agonists on major gastrointestinal disorders for type 2 diabetes mellitus: a mixed treatment comparison meta-analysis. Exp Diabetes Res 2012; DOI: 10.1155/2012/ 230624

63 Thompson AM, Trujillo JM. Dulaglutide: the newest GLP-1 receptor agonist for the management of type 2 diabetes. Ann Pharmacother 2015; 49: 351 - 359

64 Vardar B, Kizilci S. Incidence of lipohypertrophy in diabetic patients and a study of influencing factors. Diabetes Res Clin Pract 2007; 77: $231-236$

65 Von Scholten BJ, Lajer M, Goetze JP et al. Time course and mechanisms of the anti-hypertensive and renal effects of liraglutide treatment. Diabet Med 2015; 32: 343-352

66 Wang B, Zhong J, Lin $\mathrm{H}$ et al. Blood pressure-lowering effects of GLP-1 receptor agonists exenatide and liraglutide: a meta-analysis of clinical trials. Diabetes Obes Metab; 2013; 15: 737-749

$67 W u J D, X u X H, Z$, Zhu J et al. Effect of exenatide on inflammatory and oxidative stress markers in patients with type 2 diabetes mellitus. Diabetes Technol Ther 2011; 13: 143-148

68 Zinman B, Schmidt WE, Moses A et al. Achieving a clinically relevant composite outcome of an $\mathrm{HbA} 1 \mathrm{c}$ of $<7 \%$ without weight gain or hypoglycaemia in type 2 diabetes: a meta-analysis of the liraglutide clinical trial programme. Diabetes Obes Metab 2012; 14: 77-82 